\title{
In search of a UV completion of the standard model - 378,000 models that don't work
}

\author{
D. Barducci,,$^{a, b}$ M. Fabbrichesi, ${ }^{a}$ C.M. Nieto, ${ }^{a, b, c}$ R. Percacci ${ }^{a, b}$ and V. Skrinjar ${ }^{a}$ \\ ${ }^{a}$ Scuola Internazionale di Studi Superiori Avanzati (SISSA), \\ via Bonomea 265, 34136 Trieste, Italy \\ ${ }^{b}$ INFN - Sezione di Trieste, \\ via Valerio 2, 34127 Trieste, Italy \\ ${ }^{c} I C T P$, \\ Strada Costiera 11, 34151 Trieste, Italy \\ E-mail: daniele.barducci@sissa.it, marco.fabbrichesi@ts.infn.it, \\ cnieto@sissa.it, percacci@sissa.it, vedran.skrin@gmail.com
}

AbStRaCT: Asymptotically safe extensions of the Standard Model have been searched for by adding vector-like fermions charged under the Standard Model gauge group and having Yukawa-like interactions with new scalar fields. Here we study the corresponding renormalization group $\beta$-functions to next and next-to-next to leading order in the perturbative expansion, varying the number of extra fermions and the representations they carry. We test the fixed points of the $\beta$-functions against various criteria of perturbativity to single out those that are potentially viable. We show that all the candidate ultraviolet fixed points are unphysical for these models: either they are unstable under radiative corrections, or they cannot be matched to the Standard Model at low energies.

KeYwords: Beyond Standard Model, GUT

ARXIV EPRINT: 1807.05584 


\section{Contents}

1 Introduction $\quad 2$

2 Methods 4

2.1 The fixed points of the $\beta$-functions 4

2.2 Linearized flow 5

2.3 Marginal couplings $\quad 7$

2.4 Approximation schemes $\quad 7$

2.4.1 Perturbative $\beta$-functions: a digest of the literature 8

2.5 Another test of perturbativity 8

2.6 Testing fixed points with central charges 9

2.7 Standard Model matching 9

2.8 Procedure summary 10

3 The fate of the Standard Model couplings $\quad 10$

3.1 The 210 approximation scheme 11

$\begin{array}{lll}3.2 & \text { The } 321 \text { approximation scheme } & 12\end{array}$

4 Standard Model extensions $\quad 13$

$\begin{array}{lll}4.1 & \text { The } \beta \text {-functions } & 14\end{array}$

$\begin{array}{lll}4.2 & \text { Results } & 16\end{array}$

$\begin{array}{lll}\text { 4.2.1 Colorless vector-like fermions } & 16\end{array}$

4.2.2 Vector-like fermions in the fundamental of $\mathrm{SU}_{c}(3) \quad 19$

4.2.3 Vector-like fermions in higher representations of $\mathrm{SU}_{c}(3) \quad 20$

4.2.4 A model that almost works 22

4.2.5 Five benchmark models studied in the literature 24

4.2.6 Two more benchmark models studied in the literature 26

4.2.7 Combining more than one representation 26

5 Conclusions $\quad 28$

$\begin{array}{ll}\text { A Analysis of marginal couplings } & 29\end{array}$

$\begin{array}{ll}\text { B Conformal field theory and central charges } & 30\end{array}$

C All the fixed points in the 210 approximation scheme 32

D Coefficients of the NLO and NNLO $\beta$-functions 32 


\section{Introduction}

In the early days of quantum field theory, renormalizability was used as a criterion to select physically viable models. It was later understood that effective field theories can be useful and predictive in their domain of validity even if not renormalizable. Current particle physics is largely based on this paradigm. It leaves an enormous freedom. One would like to have a more restrictive framework to guide the search for physics beyond the Standard Model (BSM). Non-perturbative renormalizability, also known as asymptotic safety (AS), provides such a framework. A quantum field theory is AS if all its couplings, running along the renormalization group (RG) flow, reach a fixed point in the ultraviolet (UV) limit [1, 2]. The fixed point can be interacting or free (Gaussian). In the latter case, AS reduces to asymptotic freedom (AF). In both cases, the theory is well behaved and predictive at all energies. UV completeness is by itself a rather abstract notion, being untestable in practice. The real bonus of AS is that when a suitable fixed point exists, typically there are only a finite number of relevant directions that can be used to reach it in the UV. This greatly restricts the infrared physics.

While AF theories have been studied in great detail and for a long time, work on AS models for particle physics has only begun quite recently. For some early references based on the use of the functional renormalization group see [3-8]. A breakthrough came with the work of Litim and Sannino, who constructed gauge-Yukawa systems admitting interacting fixed points that are under perturbative control [9]. In these models the fixed point arises from a cancellation between one- and two-loop terms in the $\beta$-functions. The crucial ingredient is the Veneziano limit, providing the small expansion parameter

$$
\epsilon=\frac{N_{f}}{N_{c}}-\frac{11}{2},
$$

where $N_{c}$ and $N_{f}$ are the numbers of colors and flavors respectively. It is reasonable to expect that there may also exist AS models for finite values of $\epsilon$. General conditions for the existence of such fixed points have been discussed in $[9,10]$. Applications of these ideas to BSM physics have appeared [11-19].

The Standard Model (SM) by itself is not AS because of the Landau pole in the U(1) gauge coupling [20, 21] and the uncertain fate of the Higgs quartic interaction [22]. The Landau pole can only be avoided by assuming that the gauge coupling is identically zero at all energies. This is known as the triviality problem.

Can the SM be turned into an AS theory by extending its matter content? The simplest (and most studied) extensions consist of multiple generations of vector-like fermions carrying diverse representations of the SM gauge group. The choice of vector-like fermions is motivated by their not giving rise to gauge anomalies and their masses being technically natural. The authors of [15] have studied the $\beta$-functions to two-loop order in the simplified case of $\mathrm{SU}(3) \times \mathrm{SU}(2)$ gauge interactions and a Yukawa-like interaction among the vectorlike fermions. They find several UV fixed points, which they match to the low-energy SM in a number of benchmark cases. In a parallel development, the authors of $[16,17]$ studied AS for the full SM gauge group, again extended by vector-like fermions, by means of a re- 
summation of the perturbative series of the $\beta$-functions. They find several UV fixed points, which however cannot be matched to the low-energy SM in a consistent manner [17].

To move forward in this program, we report our results for a large class of models based on the $\mathrm{SM}$ matter content and with $\mathrm{SU}_{c}(3) \times \mathrm{SU}_{L}(2) \times U_{Y}(1)$ gauge interactions, but retaining only the top Yukawa coupling and the Higgs quartic self-interaction; in addition, the models contain fermions that are coupled to the SM gauge fields via vector currents and have Yukawa interactions with a new set of scalar fields. The restriction to the top Yukawa makes the form of the $\beta$-functions more manageable - and is in line with earlier investigations. The models differ in the number of copies of the vector-like fermions and in the representation of the gauge groups that they carry.

In contrast to $[16,17]$ we do not use resummed $\beta$-functions. Instead, we compare the results of the two-loop (NLO) gauge $\beta$-functions considered so far in the literature with the three loop results (NNLO). As explained in section 2 , the $\beta$-functions for the Yukawa and scalar couplings are retained always at one- and two-loops less than the gauge couplings, respectively. By comparing the results at these two different approximation schemes, we are able to assess the impact of radiative corrections quantitatively and therefore to decide whether a given fixed point is within the perturbative domain or not. This selection is supported by other tests of perturbativity that the fixed points must satisfy, as discussed in sections 2.5 and 2.6 .

The core of our work consists of a systematic search of reliable fixed points in a large grid parameterized by the value of $N_{f}$ (the number of vector-like fermions) and their $\mathrm{SU}(3)_{c} \times \mathrm{SU}(2)_{L} \times \mathrm{U}(1)_{Y}$ quantum numbers (this grid is defined precisely in section 4). We first find all the simultaneous zeroes of all the $\beta$-functions for each model in the grid. We then test each fixed point thus found against two conditions:

- The fixed point must occur in a region in which the perturbative expansion is reliable. At the very least, this implies that it must be reasonably possible to trace its value at the NNLO back to that of the NLO. We see a posteriori that this can be done only when the values of the couplings and of the scaling exponents (the eigenvalues of the linearized expansion around the fixed point) are sufficiently small and the fixed point satisfies all the criteria introduced in section 2 .

- The fixed point can be connected to the SM at low energy. In general this would require a delicate numerical analysis of the trajectories emanating from it. However, we find that a rough necessary condition is sufficient for our purposes: the fixed point must not have any coupling that is zero and irrelevant, because such couplings must be identically zero at all scales to avoid Landau poles.

As we shall see, these two requirements taken together, while quite reasonable, are very restrictive. As a matter of fact, we are not able to identify any choice for the group representations and number of generations of the vector-like fermions that would make the extension of the SM reliably AS. This does not mean that such an extension does not exist: it only means that if such an AS extension of the SM exists, it must either be different 
from those that we have considered, or else it must have a fixed point that lies outside the reach of perturbation theory.

\section{Methods}

In this section we describe the general procedure that we follow in the rest of the paper. This allows us to motivate better the requirements (introduced in section 1 and further elaborated here) that we impose on the fixed points in order for them to be considered as physical. We recommend [23] as a general reference on RG flows.

\subsection{The fixed points of the $\beta$-functions}

Consider a theory with generic (gauge, fermion or scalar) fields and (generally dimensionful) couplings $\bar{g}_{i}$ of the interactions among them. In the study of the RG it is customary to use dimensionless couplings $g_{i}$, related to the dimensionful couplings by $g_{i}=\mu^{-d_{i}} \bar{g}_{i}, d_{i}$ being the mass dimension of $\bar{g}_{i}$. The renormalization of the theory is completely characterized by its $\beta$-functions

$$
\beta_{i}\left(g_{j}\right) \equiv \mu \frac{d g_{i}}{d \mu}
$$

where $\mu$ is the sliding scale of the quantum theory. A fixed point of this theory, denoted $g_{j}^{*}$, is defined by the vanishing of the $\beta$-functions of all couplings:

$$
\beta_{i}\left(g_{j}^{*}\right)=0 .
$$

When the couplings $g_{j}$ assume the values $g_{j}^{*}$, the renormalization of the quantum theory stops. In general, a given fixed point can be reached either in the UV or in the IR limit, depending on the direction of the approaching trajectory. Notice that the familiar distinction between UV and IR fixed points is only meaningful when there is a single coupling in the theory.

The $\beta$-function of a single coupling is independent of the gauge choice in dimensional regularization. It is regularization scheme-independent up to NLO. If there are several couplings running together, their $\beta$-functions depend on the scheme already at the NLO [24]. There is therefore a degree of ambiguity in the position of the fixed points we are going to discuss because their position could be moved by changing the scheme. We assume that these changes are small if the fixed point is found within the perturbative regime. One should however bear in mind this problem of scheme dependence in all the discussions to follow.

In general, there are no conditions on the values of the fixed point $g_{i}^{*}$ and they could take any value. However, when we work in perturbation theory, we have to remain within its range of validity. Therefore, we demand that all the couplings be sufficiently small at the fixed point $g_{i}^{*}$. In practice this means that going to the next order of the expansion should not appreciably change the position of the fixed point as well as its other properties. We will see that this implies that the numerical value of the fixed points must satisfy the condition

$$
\alpha_{i}^{*} \equiv\left(\frac{g_{i}^{*}}{4 \pi}\right)^{2}<1,
$$


in addition to being positive. Notice that in eq. (2.3), and in what follows, the definition of the coupling $\alpha$ follows a convention widely adopted in the AS literature which however differs from the usual one by an additional factor $4 \pi$ in the denominator.

The condition in eq. (2.3) would suffice to keep the perturbative expansion within its limits of validity if the coefficients in the perturbative expansions were of the same order and not too large. If they are not, the condition in eq. (2.3) should be strengthened and only smaller values allowed to prevent terms of higher order being more important than those at the lower order.

As we shall see, this is the case for many of the fixed points we discuss. As a matter of fact, many of the fixed points discussed in the literature are due to a cancellation between the first two orders in the perturbative expansion of the $\beta$-functions. This is acceptable only if the higher terms in the perturbative expansion are then more and more suppressed. This is the main motivation for going to three-loop order in the gauge $\beta$-functions.

\subsection{Linearized flow}

Once we have a candidate fixed point, we can study the flow in its immediate neighborhood. We move away from the fixed point and study what happens when we shift the couplings by a small amount $y_{i} \equiv g_{i}-g_{i}^{*}$. To this end, we linearize the $\beta$-functions as

$$
\frac{d y_{i}}{d t}=M_{i j} y_{j}
$$

where $M_{i j} \equiv \partial \beta_{i} / \partial g_{j}$ is referred to as the stability matrix. Next, we diagonalize the linear system by going to the variables $z_{i}=\left(S^{-1}\right)_{i j} y_{j}$, defined by the equation

$$
\left(S^{-1}\right)_{i j} M_{j l} S_{l n}=\delta_{i n} \vartheta_{n},
$$

so that the $\beta$-functions and their solutions are in the simplified form

$$
\frac{d z_{i}}{d t}=\vartheta_{i} z_{i} \quad \text { and } \quad z_{i}(t)=c_{i} e^{\vartheta_{i} t}=c_{i}\left(\frac{\mu}{\mu_{0}}\right)^{\vartheta_{i}} .
$$

From the expression of $z_{i}$ as functions of $\mu$, we see that there are different situations depending on the sign of $\vartheta_{i}$ :

- For $\vartheta_{i}>0$, as we increase $\mu$ we move away from the fixed point and $z_{i}$ increases without control; the direction $z_{i}$ is said to be irrelevant.

- If $\vartheta_{i}<0$, as we increase $\mu$ we approach the fixed point; the direction $z_{i}$ is called a relevant direction.

- If $\vartheta_{i}=0$, we do not know the fate of $z_{i}$ and we have to go beyond the linear order as explained below; the direction $z_{i}$ is called marginal in this case.

The notion of relevance/irrelevance is independent of the direction of the flow and of the choice of basis. AS theories correspond to trajectories lying on the surface whose tangent space at the fixed point is spanned by the relevant directions. This tangent space 


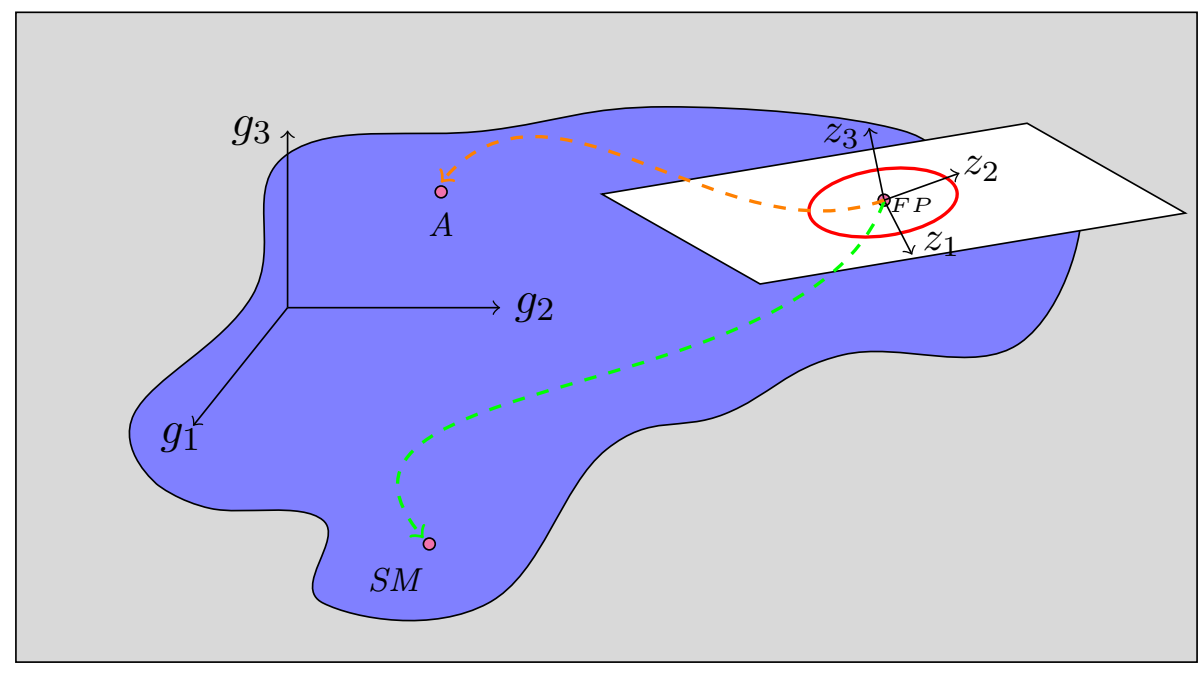

Figure 1. Theory space of couplings $g_{i}$ where only 3 axes are shown for simplicity. For a given fixed point we show the UV safe surface (blue region), the approximated UV critical surface around the fixed point (white plane), the new set of coordinates $z_{i}$, a small region of possible initial points for the flow (red circle) and two UV safe trajectories ending at a given matching scale $\mathcal{M}$ (green and orange dashed lines, the former going to the SM, the latter going to a different IR physics A).

is shown in figure 1 as a white plane. In the same figure we depict the full UV critical surface in blue.

The eigenvalues $\vartheta_{i}$ have the property of being universal quantities - meaning that they are invariant under a general transformation in the space of couplings. In perturbation theory, they cannot take any arbitrary value, there are restrictions on their size. We know that in general the $\beta$-function for $g_{i}$ has the form

$$
\beta_{i}=-d_{i} g_{i}+\beta_{i}^{q}\left(g_{j}\right)
$$

where $\beta_{i}^{q}$ encodes the pure quantum contributions to the $\beta$-functions. Therefore, the stability matrix is given by

$$
M_{i j}=-d_{i} \delta_{i j}+\frac{\partial \beta_{i}^{q}}{\partial g_{j}}
$$

which is equal to the classical scaling exponent plus quantum corrections. Then, the quantity $-\vartheta_{i}$ represents the full scaling dimension of the coupling $\bar{g}_{i}$. If we want to remain in perturbation theory, we should demand that the scaling dimension be small. In the cases we consider, where all the couplings have $d_{i}=0$, this means that

$$
\left|\vartheta_{i}\right|<O(1)
$$

There is a degree of arbitrariness about where exactly one should set this bound. In our study, we look at the scaling dimensions for the models under examination and set the bound in the first gap in the distribution of their $O(1)$ values. 


\subsection{Marginal couplings}

If one of the eigenvalues is equal to zero, the linear approximation does not give us information about the RG behaviour in the direction associated with it. Then we have to go further in the expansion [25]. At second order in the couplings $y_{i}$, the $\beta$-functions take the form

$$
\frac{d y_{i}}{d t}=M_{i j} y_{j}+P_{i j k} y_{j} y_{k}, \quad \text { where } \quad P_{i j k}=\frac{\partial^{2} \beta_{i}}{\partial g_{j} \partial g_{k}} .
$$

The structure of these quadratic flows is quite complicated to describe in full generality, with the fate of a specific trajectory depending strongly on the position of the initial point in the neighborhood of the fixed point.

However, marginal couplings do not generally occur for a fully interacting fixed point: they can always be identified with some coupling that is itself zero at the fixed point. We show in appendix A that the structure of the $\beta$-functions is such that the flow of the marginal couplings near the fixed point is of the form

$$
\frac{d y_{i}}{d t}=P_{i i i} y_{i}^{2}
$$

(no summation implied). Our flows will always be written in terms of the $\alpha_{i}$, which are bound to be positive. Therefore, marginal directions $y_{i}=\alpha_{i}$ with $P_{i i i}<0$ are UV attractive and are called marginally relevant (a well-known example being the QCD gauge coupling) while those with $P_{i i i}>0$ are UV repulsive and are called marginally irrelevant. Altogether, the UV critical surface is thus spanned by the relevant and marginally relevant directions.

\subsection{Approximation schemes}

The perturbative $\beta$-functions of the SM and its extensions have a natural hierarchy originating from the Weyl consistency conditions [26-30]:

$$
\frac{\partial \beta^{j}}{\partial g_{i}}=\frac{\partial \beta^{i}}{\partial g_{j}} .
$$

In these relations the indices on the $\beta$-functions have to be raised or lowered by means of a metric, which itself depends on different orders of the couplings. Therefore, a consistent solution of eq. (2.12) relates different orders in the perturbative expansion and indicates that the gauge couplings must have the highest order in the loop expansion, while the Yukawa coupling must be computed at one order less and the quartic interaction one further order less. This leaves us in practice with two approximations for the running of the couplings:

- the 210 approximation scheme, in which the gauge couplings are renormalized at the two-loop order (NLO), the Yukawa coupling only at one-loop order (LO) and the quartic interaction is not renormalized; and

- the 321 approximation scheme, in which the gauge couplings are renormalized at the three-loop order (NNLO), the Yukawa coupling at two-loop order (NLO) and the quartic interaction at one-loop order (LO). 
Note therefore that in the 210 approximation scheme a fixed point is defined by vanishing gauge and Yukawa $\beta$-functions, whereas in the 321 approximation scheme also the scalar quartic $\beta$-function must be zero.

By comparing the two approximations it is possible to test the stability of the fixed point against radiative corrections and the overall reliability of the perturbative computation.

Other approximation schemes are also possible, for example retaining all $\beta$-functions at the same order or keeping only the gauge $\beta$-functions one order higher than the others. These different choices do not satisfy eq. (2.12). They are analysed in [31] where their respective merits (and shortcomings) are discussed.

\subsubsection{Perturbative $\beta$-functions: a digest of the literature}

The perturbative study of the $\beta$-functions of the SM, together with some of its possible extensions, has been a collective endeavor covering many years. We collect here the main stepping stones in this ongoing computation.

The one-loop (LO) $\beta$-function for a non-abelian gauge group was computed in the classic papers [32] and [33] where AF was discovered. The LO $\beta$-function for the Yukawa coupling was presented in [34] and that for the quartic Higgs interaction in [35]. The twoloop (NLO) $\beta$-functions for the gauge groups have been calculated in [36-39], those for the Yukawa couplings in [40-42] and that for the quartic Higgs interaction in [42-44]. The case of the SM has been discussed in [45]. Mistakes in some of these results were corrected in $[46,47]$ where they were also generalized to arbitrary representations of non-simple groups. The three-loop (NNLO) $\beta$-functions of a gauge theory with simple groups were given partially in [48], then in [49]. The full NNLO $\beta$-functions for the SM were presented in [50] and those for generic representations of non-simple gauge groups in [51]. In this last paper, some contributions from the Yukawa and quartic Higgs interactions were not included. For these terms we have used currently unpublished results of L. Mihaila [52]. The NNLO $\beta$-functions for the Yukawa and quartic Higgs couplings were partially computed in $[53]$ and fully in $[54,55]$. We will not need them here.

\subsection{Another test of perturbativity}

Besides the smallness of the couplings themselves, there is another simple test that we use to assess whether a fixed point is in the perturbative domain.

Let us write the $\beta$-functions of the gauge couplings $\alpha_{i}$ in the schematic form

$$
\beta_{i}=\left(A^{(i)}+B_{r}^{(i)} \alpha_{r}+C_{r s}^{(i)} \alpha_{r} \alpha_{s}\right) \alpha_{i}^{2},
$$

where $A, B$ and $C$ are the one-, two- and three-loops coefficients. At a fixed point we can split each beta function in the following way

$$
0=\beta_{i}=A_{*}^{(i)}+B_{*}^{(i)}+C_{*}^{(i)},
$$

where $A_{*}^{(i)}=A^{(i)} \alpha_{i *}^{2}, B_{*}^{(i)}=B_{r}^{(i)} \alpha_{r *} \alpha_{i *}^{2}$ and $C_{*}^{(i)}=B_{r s}^{(i)} \alpha_{r *} \alpha_{s *} \alpha_{i *}^{2}$. When we insert the values of the fixed point calculated in the 321 approximation scheme, we expect the three 
contributions to be ordered as $\left|C_{*}^{(i)}\right|<\left|B_{*}^{(i)}\right|<\left|A_{*}^{(i)}\right|$, or equivalently

$$
\rho_{i}<\sigma_{i}<1, \quad \text { where } \rho_{i}=\left|C_{*}^{(i)} / A_{*}^{(i)}\right| \text { and } \sigma_{i}=\left|B_{*}^{(i)} / A_{*}^{(i)}\right| .
$$

Note that in this case $\rho+\sigma=1$. In principle it might also happen that the sum of the oneand three-loop terms cancel the two-loop term, i.e. $\sigma-\rho=1$. We shall see that this does not happen. Alternatively, if we insert in (2.13) the fixed point values of the 210 approximation scheme, $\beta_{i}$ will not be zero. The first two terms in (2.14) will cancel, but we still expect $\rho_{i}<1$. In the following, when we report results in the 210 approximation scheme, we give the values of $\rho_{i}$ defined at the 210 fixed point and when we report results in the 321 approximation scheme, we give the values of $\rho_{i}$ and $\sigma_{i}$ defined at the 321 approximation scheme fixed point.

\subsection{Testing fixed points with central charges}

At a fixed point the theory is a conformal field theory (CFT). As explained in appendix B, one can estimate the size of the relative changes of the central charges of the CFT to decide whether a fixed point is within the domain of perturbation theory. These relative changes are obtained in terms of the function $a=a_{\text {free }}+a_{q}$ ( $a_{q}$ refers to the contribution of quantum corrections) and of the $c$-function as

$$
\delta a \equiv \frac{a-a_{\text {free }}}{a_{\text {free }}}=\frac{a_{q}}{a_{\text {free }}} \quad \text { and } \quad \delta c \equiv \frac{c-c_{\text {free }}}{c_{\text {free }}}=\frac{c_{q}}{c_{\text {free }}} .
$$

If $\delta a$ or $\delta c$ become smaller than -1 the fixed point is unphysical because it cannot correspond to a CFT (since $c>0$ and $a>0$ are guaranteed for CFT). A fixed point for which $\delta c$ or $\delta a$ is of order 1 should be discarded as well since quantum corrections are then comparable in size to the free-theory contribution.

The central charges in the 210 approximation scheme can be easily computed by embedding the models in the general gauge-Yukawa Lagrangian of [56]. Computation in the 321 approximation scheme is significantly more complicated due to a major increase in complexity of the Zamolodchikov metric. We do not pursue the 321 computation for that reason and also because the results in the 210 approximation scheme are enough to confirm that our other perturbativity criteria are compatible with the CFT tests.

\subsection{Standard Model matching}

Once we have an understanding of the fixed point structure - and the conditions on the couplings $\alpha_{i}$ and the scaling exponents $\vartheta_{i}$ are satisfied - there remains to be found the trajectory connecting a given fixed point to the SM value of the coupling at some IR scale. This is accomplished in the following manner.

We are going to introduce BSM particles with a value $M$ of their mass that makes them undetectable at present colliders. We run all the SM couplings to this common RG scale $M$, using the SM $\beta$-functions. This defines the target for the flow to the IR from the UV fixed point. Then, the BSM RG flow is started from a point belonging to the UV critical surface, infinitesimally close to the fixed point (red circle in figure 1). This 
guarantees that, to high precision, the flow towards the UV ends at the fixed point. The system is then allowed to flow by means of the full $\beta$-functions of the theory towards the IR. The initial point of the flow is varied until, ideally, the trajectory hits exactly the target SM values.

For most of the models that we consider, this laborious procedure is not necessary. For all the fixed points that can be regarded as being in the perturbative domain, the hypercharge is zero at the fixed point and is also a marginally irrelevant coupling. This means that in order to reach the fixed point in the UV limit, the hypercharge must be zero at all energies. All other trajectories have a Landau pole. These models are thus excluded by a version of the triviality problem.

\subsection{Procedure summary}

Given a model, we first look for all the fixed points of the $\beta$-functions. Since the $\beta$-functions are given in the form of a Taylor expansion, some of their zeroes will be mere artifacts of the expansion. In practice, these we discard by requiring stability under radiative corrections. Then, in order to select the fixed points that have a chance of being physical, we demand that they can be matched to the SM at low energy.

We begin by analyzing the fixed points of the 210 approximation scheme. In the first step, we retain only those fixed points that can be reasonably assumed to be within the perturbative regime, that is, those for which the couplings and the scaling exponents satisfy the bounds in eq. (2.3) and eq. (2.9). We use the criteria discussed in sections 2.5 and 2.6 to confirm that these bounds are indeed reasonable indicators of radiative stability.

We then compare with the results of the same analysis in the 321 approximation scheme. We retain only those fixed points that can be reasonably identified in both approximations. Their number is quite small. We find that the identification is only possible if the couplings and scaling exponents are sufficiently small.

Finally, for the fixed points that are radiatively stable in the sense just described, we look for the possibility of matching to the SM at low energy. If all these conditions are satisfied, we have an acceptable fixed point. Otherwise, the fixed point is rejected.

\section{The fate of the Standard Model couplings}

The running of the SM couplings, when extended to high energies, presents two important features: partial gauge coupling unification and a Landau pole in the abelian gauge coupling. Since this singularity appears beyond the Planck scale, where gravitational effects are important, it might well happen that there will be no divergence and that all couplings are well-behaved once we consider a full theory of gravity and matter. Nevertheless, it is interesting to investigate whether such infinities could be avoided within the matter sector. This study will nicely illustrate our procedure by means of the familiar case of the SM.

Throughout this paper, we shall consider a simplified version of the SM where only the top-Yukawa coupling $y_{t}$ is retained. The remaining Yukawa couplings are set to zero. For simplicity we will keep calling this the SM. However, we stress that the degrees of freedom that enter the flow are not only those of the top quark but the full SM matter content 


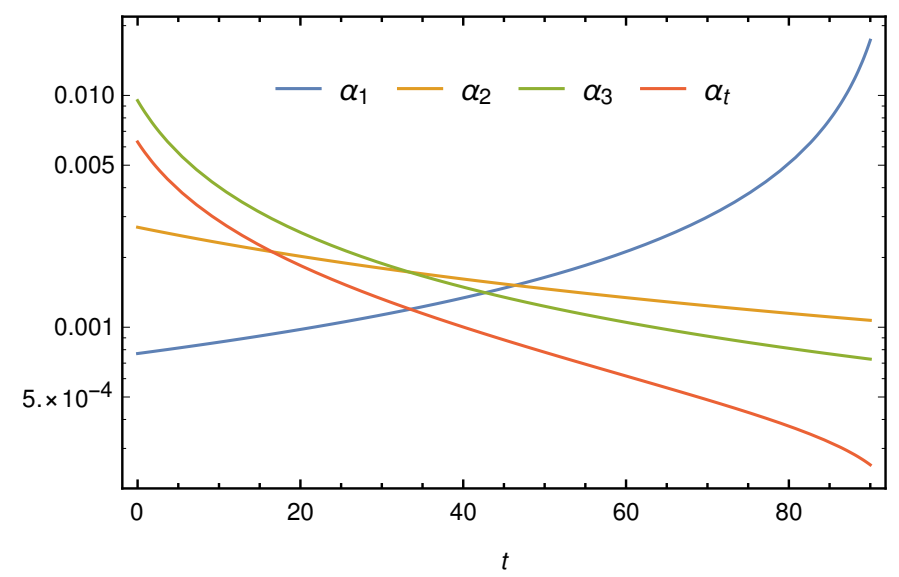

Figure 2. Running of the gauge couplings $\alpha_{i}$ and Yukawa $\alpha_{t}$ for the SM in the 321 approximation scheme. On the horizontal axis $t=\ln \left(\mu / M_{Z}\right)$. Just above $t \simeq 40$ the three gauge couplings come close together. At larger values of $t, \alpha_{1}$ begins its ascent towards the Landau pole.

(i.e., the number of fermions that enters into the 1-loop coefficient of the gauge $\beta$-functions counts all the quarks and leptons).

The first question is whether the $\beta$-functions of the SM have fixed points. This does not happen with the LO beta functions. In figure 2 we show the running of the couplings toward the (quasi)-unification point and the beginning of the ascent of the coupling $\alpha_{1}$ toward the Landau pole.

\subsection{The 210 approximation scheme}

We then consider the beta functions in the 210 approximation scheme (NLO in the gauge couplings), which are given by

$$
\begin{aligned}
& \beta_{1}^{\mathrm{NLO}}=\alpha_{1}^{2}\left(\frac{41}{3}+\frac{199}{9} \alpha_{1}+9 \alpha_{2}+\frac{88}{3} \alpha_{3}-\frac{17}{3} \alpha_{t}\right), \\
& \beta_{2}^{\mathrm{NLO}}=\alpha_{2}^{2}\left(-\frac{19}{3}+3 \alpha_{1}+\frac{35}{3} \alpha_{2}+24 \alpha_{3}-3 \alpha_{t}\right), \\
& \beta_{3}^{\mathrm{NLO}}=\alpha_{3}^{2}\left(-14+\frac{11}{3} \alpha_{1}+9 \alpha_{2}-52 \alpha_{3}-4 \alpha_{t}\right), \\
& \beta_{t}^{\mathrm{LO}}=\alpha_{t}\left(-\frac{17}{6} \alpha_{1}-\frac{9}{2} \alpha_{2}-16 \alpha_{3}+9 \alpha_{t}\right),
\end{aligned}
$$

where, following the convention (2.3), we use the variables

$$
\alpha_{i}=\frac{g_{i}^{2}}{(4 \pi)^{2}} \text { for } i=1,2,3, \quad \text { and } \quad \alpha_{t}=\frac{y_{t}^{2}}{(4 \pi)^{2}} .
$$

The set of $\beta$-functions in eq. (3.1) admits several zeroes. They are given by the last column of table 12 in appendix C. However, only two of them (solutions $P_{16}$ and $P_{17}$ ) have all $\alpha_{i}$ positive. Their properties are summarized in table 1 .

Although less than 1 , the values for the couplings constants $\alpha_{i}^{*}$ are quite sizeable and we may suspect that they lie outside the perturbative domain. This suspicion is substantiated 


\begin{tabular}{|c|c|c|c|c|c|c|c|c|}
\hline & $\alpha_{1}^{*}$ & $\alpha_{2}^{*}$ & $\alpha_{3}^{*}$ & $\alpha_{t}^{*}$ & $\vartheta_{1}$ & $\vartheta_{2}$ & $\vartheta_{3}$ & $\vartheta_{4}$ \\
\hline$F P_{1}$ & 0 & 0.543 & 0 & 0 & 3.44 & -2.44 & 0 & 0 \\
$F P_{2}$ & 0 & 0.623 & 0 & 0.311 & 5.21 & 2.21 & 0 & 0 \\
\hline
\end{tabular}

Table 1. Fixed points and their scaling exponents for the SM in the 210 approximation scheme.

by looking at the linearized flow. Considering that these exponents are classically zero, we see that the quantum corrections are quite large. The fate of the marginal directions $\left(z_{3}\right.$ and $z_{4}$ ) is determined by looking at the quadratic approximation to the flow, as discussed in section 2.3 and in appendix A. We find that the third direction is marginally irrelevant while the last one is marginally relevant.

Even if we had decided to ignore the breaking of the perturbative regime and insisted on looking for trajectories connecting one of the fixed points to the IR regime, the requirement of lying on the UV critical surface would have implied that there is always a coupling that vanishes at all scales. Namely, given that $\alpha_{1}^{*}=0$, and that the $\beta$-function for $\alpha_{1}$ is proportional to a power of $\alpha_{1}$ itself, this coupling cannot run at all. In other words, the coupling $\alpha_{1}$ is frozen at zero at all scales and the $\mathrm{U}(1)$ gauge interaction is trivial. Clearly there are no physical fixed points within the SM in the 210 expansion: the problem of the Landau pole is still present even when the gauge couplings are taken at NLO.

\subsection{The 321 approximation scheme}

To check the perturbative stability of the two fixed points of the previous section, we now study the $\beta$-functions to the next order. In the 321 approximation scheme (NNLO in the gauge couplings), the $\beta$-functions take the form [30]

$$
\begin{aligned}
\beta_{1}^{\mathrm{NNLO}}= & \beta_{1}^{\mathrm{NLO}}+\alpha_{1}^{2}\left[-\frac{388613}{2592} \alpha_{1}^{2}+\frac{205}{48} \alpha_{1} \alpha_{2}+\frac{1315}{32} \alpha_{2}^{2}-\frac{274}{27} \alpha_{1} \alpha_{3}-2 \alpha_{2} \alpha_{3}+198 \alpha_{3}^{2}\right. \\
& \left.-\left(\frac{2827}{144} \alpha_{1}+\frac{785}{16} \alpha_{2}+\frac{58}{3} \alpha_{3}\right) \alpha_{t}+\frac{315}{8} \alpha_{t}^{2}+\frac{3}{2}\left(\alpha_{1}+\alpha_{2}-\alpha_{\lambda}\right) \alpha_{\lambda}\right] \\
\beta_{2}^{\mathrm{NNLO}}= & \beta_{2}^{\mathrm{NLO}}+\alpha_{2}^{2}\left[-\frac{5597}{288} \alpha_{1}^{2}+\frac{291}{16} \alpha_{1} \alpha_{2}+\frac{324953}{864} \alpha_{2}^{2}-\frac{2}{3} \alpha_{1} \alpha_{3}+78 \alpha_{2} \alpha_{3}+162 \alpha_{3}^{2}\right. \\
& \left.-\left(\frac{593}{48} \alpha_{1}+\frac{729}{16} \alpha_{2}+14 \alpha_{3}\right) \alpha_{t}+\frac{147}{8} \alpha_{t}^{2}+\frac{1}{2}\left(\alpha_{1}+3 \alpha_{2}-3 \alpha_{\lambda}\right) \alpha_{\lambda}\right] \\
\beta_{3}^{\mathrm{NNLO}}= & \beta_{3}^{\mathrm{NLO}}+\alpha_{3}^{2}\left[-\frac{2615}{108} \alpha_{1}^{2}+\frac{1}{4} \alpha_{1} \alpha_{2}+\frac{109}{4} \alpha_{2}^{2}+\frac{154}{9} \alpha_{1} \alpha_{3}+42 \alpha_{2} \alpha_{3}+65 \alpha_{3}^{2}\right. \\
& \left.-\left(\frac{101}{12} \alpha_{1}+\frac{93}{4} \alpha_{2}+80 \alpha_{3}\right) \alpha_{t}+30 \alpha_{t}^{2}\right], \\
\beta_{t}^{\mathrm{NLO}}= & \beta_{t}^{\mathrm{LO}}+\alpha_{t}\left[+\frac{1187}{108} \alpha_{1}^{2}-\frac{3}{2} \alpha_{1} \alpha_{2}-\frac{23}{2} \alpha_{2}^{2}+\frac{38}{9} \alpha_{1} \alpha_{3}+18 \alpha_{2} \alpha_{3}-216 \alpha_{3}^{2}\right. \\
& \left.+\left(\frac{131}{8} \alpha_{1}+\frac{225}{8} \alpha_{2}+72 \alpha_{3}\right) \alpha_{t}-24 \alpha_{t}^{2}-12 \alpha_{t} \alpha_{\lambda}+3 \alpha_{\lambda}^{2}\right] \\
\beta_{\lambda}^{\mathrm{LO}}= & 12 \alpha_{\lambda}^{2}-\left(3 \alpha_{1}+9 \alpha_{2}\right) \alpha_{\lambda}+\frac{9}{4}\left(\frac{1}{3} \alpha_{1}^{2}+\frac{2}{3} \alpha_{1} \alpha_{2}+\alpha_{2}^{2}\right)+12 \alpha_{t} \alpha_{\lambda}-12 \alpha_{t}^{2}
\end{aligned}
$$




\begin{tabular}{|l|c|c|c|c|c|c|c|c|c|c|}
\hline & $\alpha_{1}^{*}$ & $\alpha_{2}^{*}$ & $\alpha_{3}^{*}$ & $\alpha_{t}^{*}$ & $\alpha_{\lambda}^{*}$ & $\vartheta_{1}$ & $\vartheta_{2}$ & $\vartheta_{3}$ & $\vartheta_{4}$ & $\vartheta_{5}$ \\
\hline$F P_{1}$ & 0 & 0 & 0 & 0.297 & 0.184 & 8.32 & -2.57 & 0 & 0 & 0 \\
$F P_{2}$ & 0 & 0.120 & 0 & 0.0695 & 0.0575 & 1.46 & 1.18 & 0.495 & 0 & 0 \\
$F P_{3}$ & 0 & 0.124 & 0 & 0.333 & 0.230 & 8.82 & -2.52 & 1.38 & 0 & 0 \\
$F P_{4}$ & 0.436 & 0.146 & 0 & 0.648 & 0.450 & -27.0 & 17.3 & -7.85 & 2.19 & 0 \\
$F P_{5}$ & 0.433 & 0 & 0 & 0.573 & 0.377 & -25.6 & 15.7 & -6.85 & 0 & 0 \\
\hline
\end{tabular}

Table 2. Fixed points and their scaling exponents for the SM in the 321 approximation scheme.

where the quartic Higgs coupling

$$
\alpha_{\lambda}=\frac{\lambda}{(4 \pi)^{2}}
$$

is no longer unrenormalized.

Due to the higher order of the equations, there are more fixed points than the two found in the 210 approximation scheme. They are listed in table 2.

Consistently with the discussion in the case of the 210 approximation scheme, neither the couplings nor the exponents are small. Moreover, it is not possible to recognize among the new fixed points those of the 210 approximation scheme: the values change dramatically, contrary to what would be expected in a well-behaved perturbative expansion.

That there is a problem is confirmed by looking at the criteria of perturbativity introduced in section 2.5. In the 210 approximation scheme, for the two fixed points of table 1 , we have $B_{*}^{(2)}=1.87$ and $B_{*}^{(2)}=2.46$, respectively, while $C_{*}^{(2)}=32.7$ and $C_{*}^{(2)}=53.9$, respectively. For both fixed points the ratio $\rho_{2}$ is of order 10 , grossly violating the bound (2.15). It therefore appears that we are outside the domain where perturbation theory can be trusted.

We conclude that the SM (at least in the simplified form considered here) does not have a physical fixed point within perturbation theory. In the next section, we study a family of models that represents the simplest extension to the SM content with the potential of generating new fixed points.

\section{Standard Model extensions}

In this section, we consider (minimal) extensions of the SM by adding new matter fields charged under the $\mathrm{SM}$ group $\mathrm{SU}_{c}(3) \times \mathrm{SU}_{L}(2) \times U_{Y}(1)$. The gauge sector is not modified. Following $[9,10,15,57]$, we take $N_{f}$ families of vector-like fermions minimally coupled to the SM. The idea is to consider a new type of Yukawa interactions among the vectorlike fermions such that their contribution generates new zeros in the gauge $\beta$-functions. Accordingly, new scalar fields must be included as well. These scalars are taken to be singlets of the SM group while the fermions carry the representations $R_{3}$ under $\mathrm{SU}_{c}(3)$, $R_{2}$ under $\mathrm{SU}_{L}(2)$, and have hypercharge $Y$ of the gauge group $U_{Y}(1)$. Denoting $S_{i j}$ the matrix formed with $N_{f}^{2}$ complex scalar fields, the Lagrangian characterizing this minimal BSM extension is

$$
\mathcal{L}=\mathcal{L}_{S M}+\operatorname{Tr}(\bar{\psi}(i \not D-M) \psi)+\operatorname{Tr}\left(\partial_{\mu} S^{\dagger} \partial_{\mu} S\right)-y \operatorname{Tr}\left(\bar{\psi}_{L} S \psi_{R}+\bar{\psi}_{R} S^{\dagger} \psi_{L}\right) .
$$


In eq. (4.1), $\mathcal{L}_{S M}$ stands for the SM lagrangian, $y$ is the BSM Yukawa coupling, which we assume to be the same for all fermions, the trace sums over the SM representation indices as well as the flavour indices, and we have decomposed $\psi$ as $\psi=\psi_{L}+\psi_{R}$ with $\psi_{R / L}=\frac{1}{2}\left(1 \pm \gamma_{5}\right) \psi$. We neglect the role of quartic self interactions of the scalars $S_{i j}$ as well as portal couplings of the latter to the Higgs sector.

This extension of the SM is simple enough to allow explicit computations while giving rise to new features in the RG flow. The vector-like fermions are a proxy for more elaborated extensions; they do not introduce gauge anomalies and do not induce a large renormalization of the Higgs mass: they are technically natural.

\subsection{The $\beta$-functions}

Within the model defined by the Lagrangian (4.1), we look for fixed points satisfying the requirements discussed in section 2.8. We start the analysis in the 210 approximation scheme and write the $\beta$-functions of the system (4.1) in terms of the quantities in eq. (3.2) augmented by the new coupling $\alpha_{y}=y^{2} /(4 \pi)^{2}$.

In the following, as in section 3, we keep only the top-Yukawa coupling. The $\beta$-functions will depend on the dimensions of the fermion representations $d$, their Casimir invariants $C$ and Dynkin indices $S$, which are defined in general as

$$
\begin{aligned}
d_{R_{2}} & =2 \ell+1, & d_{R_{3}} & =\frac{1}{2}(p+1)(q+1)(p+q+2), \\
C_{F}^{(2)} & =C_{R_{2}}=\ell(\ell+1), & C_{F}^{(3)} & =C_{R_{3}}=p+q+\frac{1}{3}\left(p^{2}+q^{2}+p q\right), \\
S_{F}^{(2)} & =S_{R_{2}}=\frac{d_{R_{2}} C_{R_{2}}}{3}, & S_{F}^{(3)} & =S_{R_{3}}=\frac{d_{R_{3}} C_{R_{3}}}{8} .
\end{aligned}
$$

Here, $\ell=0,1 / 2,1,3 / 2, \ldots$ denotes the highest weight of $R_{2}$, and $(p, q)$ (with $p, q=$ $0,1,2 \ldots)$ the weights of $R_{3}$.

In the 210 approximation scheme, the $\beta$-functions are given by $[39,41,43,46]$

$$
\begin{aligned}
\beta_{1}^{\mathrm{NLO}} & =\left(B_{1}+M_{1} \alpha_{1}+H_{1} \alpha_{2}+G_{1} \alpha_{3}-D_{1} \alpha_{y}-\frac{17}{3} \alpha_{t}\right) \alpha_{1}^{2}, \\
\beta_{2}^{\mathrm{NLO}} & =\left(-B_{2}+M_{2} \alpha_{2}+H_{2} \alpha_{1}+G_{2} \alpha_{3}-D_{2} \alpha_{y}-3 \alpha_{t}\right) \alpha_{2}^{2}, \\
\beta_{3}^{\mathrm{NLO}} & =\left(-B_{3}+M_{3} \alpha_{3}+H_{3} \alpha_{1}+G_{3} \alpha_{2}-D_{3} \alpha_{y}-4 \alpha_{t}\right) \alpha_{3}^{2}, \\
\beta_{t}^{\mathrm{LO}} & =\left(9 \alpha_{t}-\frac{17}{6} \alpha_{1}-\frac{9}{2} \alpha_{2}-16 \alpha_{3}\right) \alpha_{t}, \\
\beta_{y}^{\mathrm{LO}} & =\left(T \alpha_{y}-F_{1} \alpha_{1}-F_{2} \alpha_{2}-F_{3} \alpha_{3}\right) \alpha_{y},
\end{aligned}
$$

where we have included the gauge and matter contributions in the coefficients $B_{i}, M_{i}, H_{i}$, $G_{i}$ and $D_{i}$, for $i=1,2,3$. These coefficient are expressed in terms of $d_{R_{2}}, d_{R_{3}}, C_{R_{2}}, C_{R_{3}}$, $S_{R_{2}}, S_{R_{3}}, Y$ and $N_{f}$ as follows. For the diagonal and mixing gauge contributions to the 
gauge $\beta$-functions we have

$$
\begin{aligned}
B_{1} & =\frac{41}{3}+\frac{8}{3} N_{f} Y^{2} d_{R_{2}} d_{R_{3}}, & M_{1} & =\frac{199}{9}+8 Y^{4} N_{f} d_{R_{2}} d_{R_{3}}, \\
H_{1} & =9+8 Y^{2} N_{f} C_{R_{2}} d_{R_{2}} d_{R_{3}}, & G_{1} & =\frac{88}{3}+8 N_{f} Y^{2} C_{R_{3}} d_{R_{2}} d_{R_{3}}, \\
B_{2} & =\frac{19}{3}-\frac{8}{3} N_{f} S_{R_{2}} d_{R_{3}}, & M_{2} & =\frac{35}{3}+4 N_{f} S_{R_{2}} d_{R_{3}}\left(2 C_{R_{2}}+\frac{20}{3}\right), \\
H_{2} & =3+8 N_{f} Y^{2} S_{R_{2}} d_{R_{3}}, & G_{2} & =24+8 N_{f} S_{R_{2}} C_{R_{3}} d_{R_{3}}, \\
B_{3} & =14-\frac{8}{3} N_{f} S_{R_{3}} d_{R_{2}}, & M_{3} & =-52+4 N_{f} S_{R_{3}} d_{R_{2}}\left(2 C_{R_{3}}+10\right), \\
G_{3} & =9+8 N_{f} S_{R_{3}} C_{R_{2}} d_{R_{2}}, & H_{3} & =\frac{11}{3}+8 N_{f} Y^{2} S_{R_{3}} d_{R_{2}} .
\end{aligned}
$$

For the Yukawa contribution to the gauge $\beta$-functions we have

$$
D_{1}=4 N_{f}^{2} Y^{2} d_{R_{2}} d_{R_{3}}, \quad D_{2}=\frac{1}{3} 4 N_{f}^{2} C_{R_{2}} d_{R_{2}} d_{R_{3}}, \quad D_{3}=\frac{1}{8} 4 N_{f}^{2} C_{R_{3}} d_{R_{2}} d_{R_{3}},
$$

whereas the running of the new coupling $\alpha_{y}$ is characterized by the coefficients

$$
T=2\left(N_{f}+d_{R_{2}} C_{R_{3}}\right), \quad F_{1}=12 Y^{2}, F_{2}=12 C_{R_{2}}, F_{3}=12 C_{R_{3}} .
$$

All the new contributions to the gauge couplings running are multiplied by $N_{f}$, meaning that we can go back to the SM by taking the $N_{f} \rightarrow 0$ limit.

Due to the simplicity of the $\beta$-functions to this order in perturbation theory, we can find analytic solutions of the equations $\beta_{i}^{\mathrm{NLO}}=\beta_{t}^{\mathrm{LO}}=\beta_{y}^{\mathrm{LO}}=0$ as functions of $Y, \ell, p, q$ and $N_{f}$. All these solutions are listed in table 12 and can be split in two categories according to whether they depend on the hypercharge $Y$ or not. All the latter have $\alpha_{1}^{*}=0$.

For the gauge couplings, using the variables in eq. (3.2), the $\beta$-functions in the 321 approximation scheme are given as follows

$$
\begin{aligned}
\beta_{1}^{\mathrm{NNLO}}= & \beta_{1}^{\mathrm{NLO}}+\left[-M_{11} \alpha_{1}^{2}+M_{12} \alpha_{1} \alpha_{2}-M_{13} \alpha_{1} \alpha_{3}-G_{23} \alpha_{2} \alpha_{3}+H_{11} \alpha_{2}^{2}+G_{11} \alpha_{3}^{2}\right. \\
& +\frac{315}{8} \alpha_{t}^{2}+K_{y 1} \alpha_{y}^{2}-\frac{2827}{144} \alpha_{1} \alpha_{t}-\frac{785}{16} \alpha_{2} \alpha_{t}-\frac{58}{3} \alpha_{3} \alpha_{t} \\
& \left.-\left(K_{11} \alpha_{1}+K_{12} \alpha_{2}+K_{13} \alpha_{3}\right) \alpha_{y}+\frac{3}{2}\left(\alpha_{1}+\alpha_{2}-\alpha_{\lambda}\right) \alpha_{\lambda}\right] \alpha_{1}^{2}, \\
\beta_{2}^{\mathrm{NNLO}}= & \beta_{2}^{\mathrm{NLO}}+\left[-M_{22} \alpha_{2}^{2}+M_{21} \alpha_{2} \alpha_{1}-M_{23} \alpha_{2} \alpha_{3}-G_{13} \alpha_{1} \alpha_{3}-H_{22} \alpha_{1}^{2}+G_{22} \alpha_{3}^{2}\right. \\
& +\frac{147}{8} \alpha_{t}^{2}+K_{y 2} \alpha_{y}^{2}-\frac{729}{16} \alpha_{2} \alpha_{t}-\frac{593}{48} \alpha_{1} \alpha_{t}-14 \alpha_{3} \alpha_{t} \\
& \left.-\left(K_{22} \alpha_{2}+K_{21} \alpha_{1}+K_{23} \alpha_{3}\right) \alpha_{y}+\frac{1}{2}\left(\alpha_{1}+3 \alpha_{2}-3 \alpha_{\lambda}\right) \alpha_{\lambda}\right] \alpha_{2}^{2}, \\
\beta_{3}^{\mathrm{NNLO}}= & \beta_{3}^{\mathrm{NNLO}}+\left[-M_{33} \alpha_{3}^{2}+M_{31} \alpha_{3} \alpha_{1}-M_{32} \alpha_{3} \alpha_{2}-G_{12} \alpha_{1} \alpha_{2}-H_{33} \alpha_{2}^{2}+G_{33} \alpha_{2}^{2}\right. \\
& +30 \alpha_{t}^{2}+K_{3 y} \alpha_{y}^{2}-80 \alpha_{3} \alpha_{t}-\frac{101}{12} \alpha_{1} \alpha_{t}-\frac{93}{4} \alpha_{2} \alpha_{t} \\
& \left.-\left(K_{33} \alpha_{3}+K_{31} \alpha_{1}+K_{32} \alpha_{2}\right) \alpha_{y}\right] \alpha_{3}^{2} .
\end{aligned}
$$


For the Yukawa and quartic Higgs couplings, the $\beta$-functions are given by

$$
\begin{aligned}
\beta_{t}^{\mathrm{NLO}}= & \beta_{t}^{\mathrm{LO}}+\left[-24 \alpha_{t}^{2}+3 \alpha_{\lambda}^{2}-12 \alpha_{t} \alpha_{\lambda}+\left(\frac{131}{8} \alpha_{1}+\frac{225}{8} \alpha_{2}+72 \alpha_{3}\right) \alpha_{t}\right. \\
& +\frac{1187}{108} \alpha_{1}^{2}+\frac{3}{2} \alpha_{1} \alpha_{2}-\frac{23}{2} \alpha_{2}^{2}+\frac{38}{9} \alpha_{1} \alpha_{3}+18 \alpha_{2} \alpha_{3}-216 \alpha_{3}^{2} \\
& \left.+\frac{58}{27} B_{t 1} \alpha_{1}^{2}+2 B_{t 2} \alpha_{2}^{2}+\frac{160}{9} B_{t 3} \alpha_{3}^{2}\right] \alpha_{t} \\
\beta_{y}^{\mathrm{NLO}}= & \beta_{y}^{\mathrm{LO}}+\left[(4-V) \alpha_{y}^{2}+\left(V_{1} \alpha_{1}+V_{2} \alpha_{2}+V_{3} \alpha_{3}\right) \alpha_{y}\right. \\
& \left.+W_{1} \alpha_{1}^{2}+W_{2} \alpha_{2}^{2}+W_{3} \alpha_{3}^{2}-W_{12} \alpha_{1} \alpha_{2}-W_{13} \alpha_{1} \alpha_{2}-W_{23} \alpha_{2} \alpha_{3}\right] \alpha_{y}, \\
\beta_{\lambda}^{\mathrm{LO}}= & 12 \alpha_{\lambda}^{2}-\left(3 \alpha_{1}+9 \alpha_{2}\right) \alpha_{\lambda}+\frac{9}{4}\left(\frac{1}{3} \alpha_{1}^{2}+\frac{2}{3} \alpha_{1} \alpha_{2}+\alpha_{2}^{2}\right)+12 \alpha_{t} \alpha_{\lambda}-12 \alpha_{t}^{2} .
\end{aligned}
$$

In eqs. (4.7)-(4.8), we have introduced several coefficients containing the gauge and Yukawa contributions which depend on $N_{f}$ and the group representations of the SM and new vectorlike fermions. These coefficients are given in appendix D.

It is not possible to find analytic solutions for the fixed points in the 321 approximation scheme. The system $\beta_{i}^{\mathrm{NNLO}}=\beta_{t}^{\mathrm{NLO}}=\beta_{y}^{\mathrm{NLO}}=\beta_{\lambda}^{\mathrm{LO}}=0$ must be solved numerically, separately for each given choice of $\left(N_{f}, Y, p, q, \ell\right)$. No separation between $Y$-independent and dependent solutions can be established before solving the equations.

\subsection{Results}

In order to find fixed points satisfying the conditions (2.3) and (2.9), we generate a grid in the space spanned by the quantum numbers $\left(N_{f}, \ell, Y\right)$ for three specific $\mathrm{SU}_{c}(3)$ representations: colorless $(p=q=0)$, fundamental $(p=1, q=0)$ and adjoint $(p=q=1)$. For each of these representations, we consider the following values for the number of vector-like fermions, their isospin and hypercharge: $N_{f} \in[1,300]$ in steps of size $1, \ell \in[1 / 2,10]$ and $Y \in[0,10]$ both in steps of size $1 / 2$. This amounts to 126,000 points for each representation of $\mathrm{SU}_{c}(3)$.

\subsubsection{Colorless vector-like fermions}

Colorless vector-like fermions are the least phenomenologically restricted and therefore the most attractive candidates for a successful extension of the SM. In the 210 approximation scheme we find that only the $Y$-independent set of solutions contains fixed points fulfilling the required conditions $(\alpha<1,|\vartheta|<O(1))$.

To set the precise bound on $|\vartheta|$, we plot in figure 3 the largest eigenvalues of the stability matrix. For the $Y$-independent solutions there is a gap between 2.21 and 62.6; for the $Y$-dependent solutions there are no eigenvalues less than 9.63. Accordingly, we decide to consider fixed points with $|\vartheta|<3$. In this way we probably include some fixed points that are not within perturbation theory, but we prefer to err on this side than to miss potentially interesting fixed points. In this way we discard all the $Y$-dependent fixed points since there is always an eigenvalue which is at least of order 10 . 


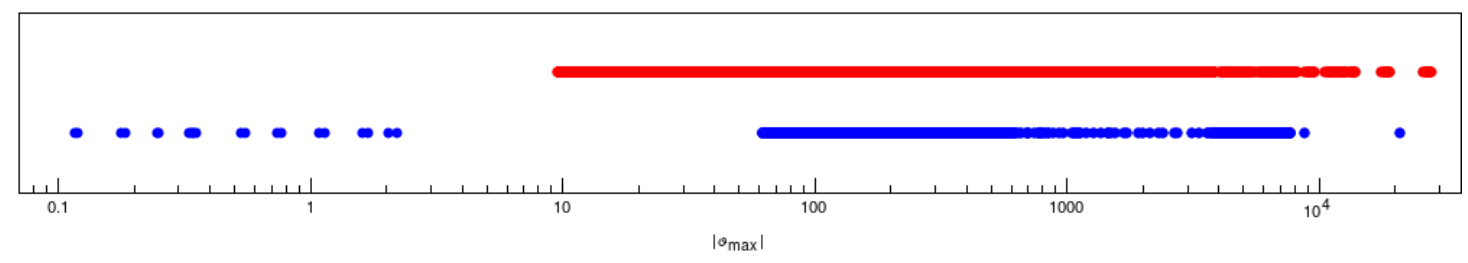

Figure 3. Distribution of the largest eigenvalues $\vartheta_{\max }$ of the stability matrix of the fixed points of the colorless models. Blue dots: eigenvalues for the $Y$-independent solutions: there is a gap between 2.21 and 62.6. Red dots: eigenvalues for the $Y$-dependent solutions: there is no gap, the eigenvalues start around 10 .

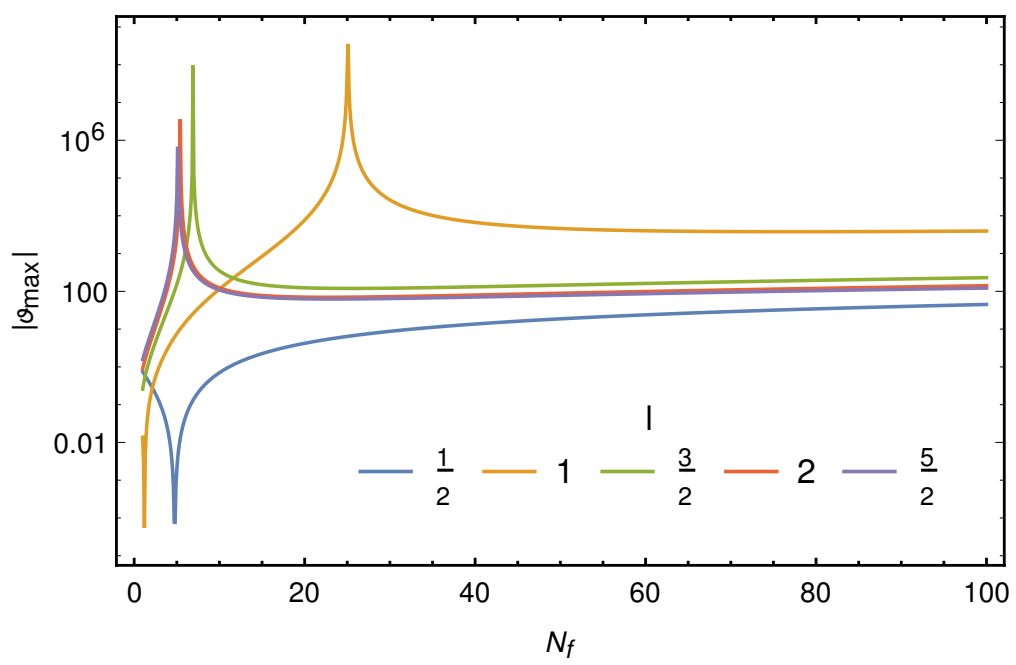

Figure 4. Behaviour of a given eigenvalue $|\vartheta|$ as a function of $N_{f}$ for several values of $\ell$ in the colorless case. The scaling dimension increases very fast with $N_{f}$, and only small values of $N_{f}, \ell$ produce $|\vartheta|<O(1)$.

After having applied all the criteria discussed in section 2 we find that, for any value of the hypercharge $Y$, the only representations producing satisfactory candidate fixed points are those collected, together with the corresponding eigenvalues, in table 3 . The eigenvalues of the stability matrix turn out to be $Y$-independent as well. We also show in table 3 the ratio $\rho_{2}$. As discussed in section 2.5, this shows how large the three-loop contribution is with respect to the two-loop contribution.

The bounds on $N_{f}$ and $\ell$ come from the behavior of the eigenvalues as functions of these parameters. If we plot one of the eigenvalues as a function of $N_{f}$ for several values of $l$, we observe that it increases very fast. From figure 4, we see that only models with small $N_{f}$ produce sufficiently small eigenvalues.

It is important to note that the large scaling dimensions of models with large $N_{f}$ frustrate the apparently promising strategy of increasing $N_{f}$ in order to increase the NLO term in the gauge $\beta$-functions to cancel the $\left(N_{f}\right.$-independent) LO term with smaller (and therefore more perturbative) values of the couplings $\alpha_{i}$.

The above selection of the viable fixed points is confirmed by the study of their CFT central charges. There are $20 Y$-independent fixed points with eigenvalues up to about 


\begin{tabular}{|c|c|c|c|c|c|c|c|c|c|c|c|c|}
\hline$\left(N_{f}, \ell\right)$ & $\alpha_{1}^{*}$ & $\alpha_{2}^{*}$ & $\alpha_{3}^{*}$ & $\alpha_{t}^{*}$ & $\alpha_{y}^{*}$ & $\vartheta_{1}$ & $\vartheta_{2}$ & $\vartheta_{3}$ & $\vartheta_{4}$ & $\vartheta_{5}$ & & $\rho_{2}$ \\
\hline \multirow{2}{*}{$\left(1, \frac{1}{2}\right)$} & 0 & 0.200 & 0 & 0 & 0.300 & 2.04 & -0.900 & 0.884 & 0 & 0 & $P_{16}$ & 3.97 \\
& 0 & 0.213 & 0 & 0.106 & 0.319 & 2.21 & 1.19 & 0.743 & 0 & 0 & $P_{17}$ & 4.33 \\
& 0 & 0.179 & 0 & 0 & 0 & -1.61 & 0.893 & -0.804 & 0 & 0 & $P_{18}$ & 3.28 \\
& 0 & 0.189 & 0 & 0.0943 & 0 & -1.70 & 1.15 & 0.697 & 0 & 0 & $P_{19}$ & 3.53 \\
\hline \multirow{2}{*}{$(1,1)$} & 0 & 0.0137 & 0 & 0 & 0.0411 & 0.333 & -0.0616 & 0.0135 & 0 & 0 & $P_{16}$ & 0.194 \\
& 0 & 0.0140 & 0 & 0.0070 & 0.0420 & 0.341 & 0.0633 & 0.0137 & 0 & 0 & $P_{17}$ & 0.198 \\
& 0 & 0.0103 & 0 & 0 & 0 & -0.247 & -0.0464 & 0.0103 & 0 & 0 & $P_{18}$ & 0.0963 \\
& 0 & 0.0105 & 0 & 0.0052 & 0 & -0.251 & 0.0473 & 0.0104 & 0 & 0 & $P_{19}$ & 0.0973 \\
\hline \multirow{2}{*}{$\left(2, \frac{1}{2}\right)$} & 0 & 0.104 & 0 & 0 & 0.117 & 1.0833 & -0.467 & 0.328 & 0 & 0 & $P_{16}$ & 1.71 \\
& 0 & 0.108 & 0 & 0.0542 & 0.122 & 1.14 & 0.525 & 0.315 & 0 & 0 & $P_{17}$ & 1.81 \\
& 0 & 0.0827 & 0 & 0 & 0 & -0.744 & -0.372 & 0.303 & 0 & 0 & $P_{18}$ & 1.19 \\
& 0 & 0.0856 & 0 & 0.0428 & 0 & -0.770 & 0.427 & 0.283 & 0 & 0 & $P_{19}$ & 1.23 \\
\hline \multirow{2}{*}{$\left(3, \frac{1}{2}\right)$} & 0 & 0.0525 & 0 & 0 & 0.0472 & 0.530 & -0.236 & 0.109 & 0 & 0 & $P_{16}$ & 0.763 \\
& 0 & 0.0543 & 0 & 0.0272 & 0.0489 & 0.552 & 0.251 & 0.109 & 0 & 0 & $P_{17}$ & 0.794 \\
& 0 & 0.0385 & 0 & 0 & 0 & -0.346 & -0.173 & 0.0897 & 0 & 0 & $P_{18}$ & 0.471 \\
& 0 & 0.0394 & 0 & 0.0197 & 0 & -0.355 & 0.182 & 0.0896 & 0 & 0 & $P_{19}$ & 0.483 \\
\hline \multirow{3}{*}{$\left(4, \frac{1}{2}\right)$} & 0 & 0.0189 & 0 & 0 & 0.0141 & 0.179 & -0.0849 & 0.0179 & 0 & 0 & $P_{16}$ & 0.246 \\
& 0 & 0.0194 & 0 & 0.0097 & 0.0146 & 0.185 & 0.0880 & 0.0182 & 0 & 0 & $P_{17}$ & 0.253 \\
& 0 & 0.0130 & 0 & 0 & 0 & -0.117 & -0.0584 & 0.0130 & 0 & 0 & $P_{18}$ & 0.141 \\
& 0 & 0.0132 & 0 & 0.0066 & 0 & -0.119 & 0.0599 & 0.0132 & 0 & 0 & $P_{19}$ & 0.143 \\
\hline
\end{tabular}

Table 3. Set of fixed points and eigenvalues for colorless vector-like fermions in the 210 approximation scheme. We highlight in green the fixed points that appear also in the 321 approximation. The labels in the second to the last last column refer to the list in table 12 . We show in the last column the ratio $\rho_{2}$ defined in eq. (2.14) knowing that in $210 A_{*}^{(2)}=B_{*}^{(2)}$.

\pm 2 . The fixed point with least variation in the central charges is that with $\left(N_{f}, \ell\right)=$ $(1,1)$, having $\delta a \simeq-0.0007$ and $\delta c \simeq 0.08$. The one with the largest change is that with $\left(N_{f}, \ell\right)=(1,1 / 2)$, having $\delta a \simeq-0.2$ and $\delta c \simeq 0.8$. All these fixed points (except for the one corresponding to $\left.\left(N_{f}, \ell, Y\right)=(1,1 / 2,0)\right)$ pass the collider bounds test (see appendix B). There are $69 Y$-dependent fixed points with eigenvalues up to \pm 10 . None of them have positive $a$ or $c$ with $\delta a$ and $\delta c$ being of $O(1)$. They should all be discarded. These results confirm our classification of the fixed points in table 3 according to the size of their eigenvalues and the ratio $\rho$.

Now that we have isolated the candidates to study, we check whether these fixed points can be connected to the SM via the RG flow. We find that $\beta_{1}$ is proportional to $\alpha_{1}^{2}$ and so, in order to avoid Landau poles, $\alpha_{1}$ has to vanishes at all energy scales. In conclusion, although we have perturbative fixed points, these cannot be matched to the SM because we know that $g_{1}$ is different from zero at the $\mathrm{TeV}$ scale.

We then perform a similar search in the 321 approximation scheme. Since we see in table 3 that the fixed point with $|\vartheta|>1$ produce a rather large $\rho_{2}$ ratio, we stick to 


\begin{tabular}{|c|c|c|c|c|c|c|c|c|c|c|c|c|c|c|}
\hline$\left(N_{f}, l\right)$ & $\alpha_{1}^{*}$ & $\alpha_{2}^{*}$ & $\alpha_{3}^{*}$ & $\alpha_{t}^{*}$ & $\alpha_{y}^{*}$ & $\alpha_{\lambda}^{*}$ & $\vartheta_{1}$ & $\vartheta_{2}$ & $\vartheta_{3}$ & $\vartheta_{4}$ & $\vartheta_{5}$ & $\vartheta_{6}$ & $\sigma_{2}$ & $\rho_{2}$ \\
\hline$(1,1)$ & 0 & 0.0096 & 0 & 0.0048 & 0 & 0.0039 & -0.244 & 0.0655 & 0.0430 & 0.0103 & 0 & 0 & 0.918 & 0.0821 \\
& 0 & 0.0119 & 0 & 0.0060 & 0.0343 & 0.0048 & 0.301 & 0.0813 & 0.0531 & 0.0134 & 0 & 0 & 0.8601 & 0.140 \\
\hline$\left(2, \frac{1}{2}\right)$ & 0 & 0.0498 & 0 & 0.0259 & 0 & 0.0211 & -0.592 & 0.382 & 0.282 & 0.200 & 0 & 0 & 0.581 & 0.418 \\
& 0 & 0.0567 & 0 & 0.0296 & 0.0734 & 0.0242 & 0.696 & 0.442 & 0.314 & 0.224 & 0 & 0 & 0.5012 & 0.499 \\
\hline$\left(3, \frac{1}{2}\right)$ & 0 & 0.0291 & 0 & 0.0148 & 0 & 0.0120 & -0.306 & 0.2080 & 0.132 & 0.0827 & 0 & 0 & 0.737 & 0.263 \\
& 0 & 0.0362 & 0 & 0.0184 & 0.0353 & 0.0150 & 0.403 & 0.262 & 0.165 & 0.100 & 0 & 0 & 0.645 & 0.354 \\
\hline \multirow{2}{*}{$\left(4, \frac{1}{2}\right)$} & 0 & 0.0117 & 0 & 0.0059 & 0 & 0.0048 & -0.112 & 0.0804 & 0.052 & 0.0130 & 0 & 0 & 0.887 & 0.113 \\
& 0 & 0.0162 & 0 & 0.0081 & 0.0125 & 0.0066 & 0.161 & 0.112 & 0.0723 & 0.0179 & 0 & 0 & 0.823 & 0.177 \\
\hline
\end{tabular}

Table 4. Fixed points and eigenvalues for colorless vector-like fermions, in the 321 approximation scheme. The last two columns give the values of the ratios $\sigma_{2}$ and $\rho_{2}$ (see 2.15).

solutions having $|\vartheta|<1$. We find that the same combinations of $N_{f}$ and $\ell$ that provide perturbative fixed points in the 210 case, also give viable solutions here. Moreover, the solutions turn out to be $Y$-independent as well.

In table 4 we show the fixed point solutions satisfying the criteria in eq. (2.3) and eq. (2.9). All the fixed points in table 4 can be traced back to fixed points that were already present in the 210 approximation scheme and listed in table 3 . Notice that for a given pair $\left(N_{f}, \ell\right)$, not all the fixed points in 210 persist. For those that do, the values of $\alpha^{*}$ and $\vartheta$ change by relatively small amount. We can then claim that the solutions given in table 4 are radiatively stable fixed points.

Unfortunately, when we look at trajectories lying on the UV critical surface, we find again that the coupling $\alpha_{1}$ must be zero at all scales in all the models. The abelian interactions suffer from the triviality problem and no matching to the SM is possible if asymptotic safety is assumed.

All these colorless models are therefore ruled out.

\subsubsection{Vector-like fermions in the fundamental of $\mathrm{SU}_{c}(3)$}

For the fundamental representation ( $p=1$ and $q=0$ or vice-versa) we follow the same procedure as before and generate 126,000 models by scanning the same grid in the $\left(N_{f}, \ell, Y\right)$ space. We split the solutions in two families depending on whether they depend on the value of their hypercharge $Y$ or not. The distribution of the largest eigenvalues given in figure 5 shows that there are no fixed points with $|\vartheta|<52.1$ for the $Y$-dependent solutions, whereas for the $Y$-independent solutions there is a gap between 10.8 and 372 . Accordingly, we eliminate all $Y$-dependent solutions and impose the bound $|\vartheta|<11$ for those that are $Y$ independent. In this way, even more than in the preceding section, we include models that are probably unreliable, but these can be eliminated at a later stage. For the $Y$-independent solutions, we find the combinations of $N_{f}$ and $\ell$ in tables 5 and 6 that generate satisfactory candidate fixed points.

This selection is confirmed by the study of the central charges for these models. Among the 49 distinct $Y$-independent fixed points with eigenvalues up to \pm 10 , all have positive $c$-function, but 6 of them have a negative $a$-function (with one more being borderline acceptable). The CFT test seems to work well here: all fixed points with reasonable 


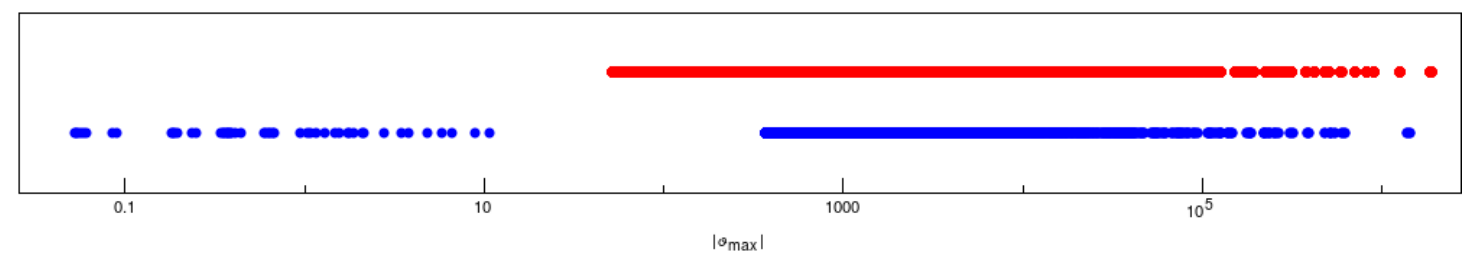

Figure 5. Distribution of the largest eigenvalues $\vartheta_{\max }$ of the stability matrix of the fixed points of the SU(3) fundamental representation. Blue dots: eigenvalues for the $Y$-independent solutions: there is a gap between 10.8 and 372. Red dots: eigenvalues for the $Y$-dependent solutions: there is no gap, the eigenvalues start at 52.1 .

critical exponents pass it, whereas the ones with relatively large exponents do not. An unexpected fact is that the separation between large and small exponents seems to be around a maximum value of $|\vartheta|$ around 3. For these perturbative and "semi-perturbative" fixed points, we also notice that the $a$-function is generically pushed toward $0\left(a_{q}<0\right)$ whereas the $c$ function is generically shifted to larger values $\left(c_{q}>0\right)$. This is why the fixed points with negative $a$-function still seem to pass the $c$-function test. If one considers $\delta c$ instead, then for most of these fixed points $\delta c>1$, but apparently not for all. Finally, if one also studies the collider bounds one finds that ten more fixed points are excluded, usually those which just barely satisfied one or both of the $a$ and $c$ tests. The collider bounds tests seem to be the most stringent.

When one tries to match these fixed points to the SM at low energies, it turns out that the abelian gauge coupling $\alpha_{1}$ must again be zero at all scales. None of these fixed points is physically viable.

In the 321 approximation scheme, there exist fixed points that can be reasonably traced back to those in the 210 approximation scheme. These solutions are shown in table 7 , where we have included only fixed points with $|\vartheta|<1$ in order to get small ratios $\rho_{i}$ and $\sigma_{i}$. However, they all have at least one coupling that has to be zero at all scales, thus preventing a proper matching to the SM.

We conclude that also all the models with the vector-like fermions in the fundamental representation of $\mathrm{SU}_{c}(3)$ cannot provide an $\mathrm{AS}$ extension to the $\mathrm{SM}$.

\subsubsection{Vector-like fermions in higher representations of $\mathrm{SU}_{c}(3)$}

For the adjoint representation (with $p=q=1$ ), the search over the same grid of values for $\left(N_{f}, \ell, Y\right)$ (and thus 126,000 further models) does not produce any fixed point within the perturbative domain. This is true both in the 210 and in the 321 approximation scheme.

In figure 6 , we show the distribution the largest eigenvalues of the stability matrix for representative couplings of the fixed points for the 210 approximation scheme. We clearly see that the eigenvalues are rather large. In fact, the minimum eigenvalue in the $Y$-independent set of solutions is 1342, while in the $Y$-dependent set is 426 .

This problem is confirmed by the study of the central charges. For the $Y$-independent fixed points we find for all fixed points $\delta a$ of $O(1000)$. Similarly, for the $Y$-dependent the 


\begin{tabular}{|c|c|c|c|c|c|c|c|c|c|c|c|c|}
\hline$\left(N_{f}, l\right)$ & $\alpha_{1}^{*}$ & $\alpha_{2}^{*}$ & $\alpha_{3}^{*}$ & $\alpha_{t}^{*}$ & $\alpha_{y}^{*}$ & $\vartheta_{1}$ & $\vartheta_{2}$ & $\vartheta_{3}$ & $\vartheta_{4}$ & $\vartheta_{5}$ & & $\rho$ \\
\hline \multirow[t]{4}{*}{$\left(1, \frac{1}{2}\right)$} & 0 & 0.0411 & 0 & 0 & 0.0264 & 0.378 & -0.185 & 0.0936 & 0 & 0 & $P_{16}$ & 0.522 \\
\hline & 0 & 0.0422 & 0 & 0.0211 & 0.0271 & 0.389 & 0.195 & 0.0936 & 0 & 0 & $P_{17}$ & 0.537 \\
\hline & 0 & 0.0385 & 0 & 0 & 0 & -0.346 & -0.173 & 0.0897 & 0 & 0 & $P_{18}$ & 0.471 \\
\hline & 0 & 0.0394 & 0 & 0.0197 & 0 & -0.355 & 0.182 & 0.0896 & 0 & 0 & $P_{19}$ & 0.483 \\
\hline \multirow[t]{2}{*}{$(1,1)$} & 0 & 0 & 0.417 & 0 & 0 & -6.67 & -6.67 & 4.17 & 0 & 0 & $P_{11}$ & 20.9 \\
\hline & 0 & 0 & 0.521 & 0 & 0.417 & 10.8 & -8.33 & 4.00 & 0 & 0 & $P_{9}$ & 31.8 \\
\hline \multirow{4}{*}{$\left(1, \frac{3}{2}\right)$} & 0 & 0 & 0.176 & 0 & 0 & -2.81 & -2.81 & 1.52 & 0 & 0 & $P_{11}$ & 5.45 \\
\hline & 0 & 0 & 0.205 & 0.365 & 0 & 3.84 & -3.28 & 1.52 & 0 & 0 & $P_{10}$ & 7.21 \\
\hline & 0 & 0 & 0.195 & 0 & 0.120 & 3.49 & -3.12 & 1.51 & 0 & 0 & $P_{9}$ & 6.60 \\
\hline & 0 & 0 & 0.232 & 0.413 & 0.143 & 4.83 & 3.72 & 1.55 & 0 & 0 & $P_{8}$ & 9.06 \\
\hline \multirow[t]{4}{*}{$(1,2)$} & 0 & 0 & 0.0982 & 0 & 0 & -1.57 & -1.57 & 0.720 & 0 & 0 & $P_{11}$ & 2.42 \\
\hline & 0 & 0 & 0.108 & 0.193 & 0 & 1.88 & -1.74 & 0.735 & 0 & 0 & $P_{10}$ & 2.88 \\
\hline & 0 & 0 & 0.105 & 0 & 0.0526 & 1.78 & -1.68 & 0.730 & 0 & 0 & $P_{9}$ & 2.73 \\
\hline & 0 & 0 & 0.117 & 0.208 & 0.0586 & 2.15 & 1.88 & 0.749 & 0 & 0 & $P_{8}$ & 3.30 \\
\hline \multirow{4}{*}{$\left(1, \frac{5}{2}\right)$} & 0 & 0 & 0.0600 & 0 & 0 & -0.960 & -0.960 & 0.360 & 0 & 0 & $P_{11}$ & 1.27 \\
\hline & 0 & 0 & 0.0646 & 0.115 & 0 & 1.08 & -1.03 & 0.371 & 0 & 0 & $P_{10}$ & 1.44 \\
\hline & 0 & 0 & 0.0632 & 0 & 0.0266 & 1.04 & -1.01 & 0.368 & 0 & 0 & $P_{9}$ & 1.39 \\
\hline & 0 & 0 & 0.0683 & 0.121 & 0.0288 & 1.18 & 1.09 & 0.380 & 0 & 0 & $P_{8}$ & 1.59 \\
\hline \multirow[t]{4}{*}{$(1,3)$} & 0 & 0 & 0.0412 & 0.0733 & 0.0150 & 0.689 & 0.660 & 0.184 & 0 & 0 & $P_{8}$ & 0.839 \\
\hline & 0 & 0 & 0.0388 & 0 & 0.0141 & 0.632 & -0.621 & 0.178 & 0 & 0 & $P_{9}$ & 0.758 \\
\hline & 0 & 0 & 0.0395 & 0.0702 & 0 & 0.647 & -0.632 & 0.180 & 0 & 0 & $P_{10}$ & 0.778 \\
\hline & 0 & 0 & 0.0372 & 0 & 0 & -0.596 & -0.596 & 0.174 & 0 & 0 & $P_{11}$ & 0.707 \\
\hline \multirow[t]{4}{*}{$\left(1, \frac{7}{2}\right)$} & 0 & 0 & 0.0221 & 0 & 0 & -0.354 & -0.354 & 0.0737 & 0 & 0 & $P_{11}$ & 0.384 \\
\hline & 0 & 0 & 0.0232 & 0.0413 & 0 & 0.376 & -0.371 & 0.0764 & 0 & 0 & $P_{10}$ & 0.415 \\
\hline & 0 & 0 & 0.0229 & 0 & 0.0073 & 0.370 & -0.366 & 0.0756 & 0 & 0 & $P_{9}$ & 0.406 \\
\hline & 0 & 0 & 0.0241 & 0.0428 & 0.0077 & 0.394 & 0.385 & 0.0784 & 0 & 0 & $P_{8}$ & 0.441 \\
\hline \multirow[t]{4}{*}{$(1,4)$} & 0 & 0 & 0.0114 & 0 & 0 & -0.182 & -0.182 & 0.0235 & 0 & 0 & $P_{11}$ & 0.182 \\
\hline & 0 & 0 & 0.0118 & 0.0210 & 0 & 0.191 & -0.189 & 0.0235 & 0 & 0 & $P_{10}$ & 0.195 \\
\hline & 0 & 0 & 0.0117 & 0 & 0.0033 & 0.188 & -0.187 & 0.0233 & 0 & 0 & $P_{9}$ & 0.191 \\
\hline & 0 & 0 & 0.0122 & 0.0217 & 0.0035 & 0.197 & 0.195 & 0.0242 & 0 & 0 & $P_{8}$ & 0.205 \\
\hline \multirow[t]{4}{*}{$\left(1, \frac{9}{2}\right)$} & 0 & 0 & 0.0033 & 0 & 0 & -0.0530 & -0.0530 & 0.0022 & 0 & 0 & $P_{11}$ & 0.0495 \\
\hline & 0 & 0 & 0.0034 & 0.0061 & 0 & 0.0550 & -0.0549 & 0.0023 & 0 & 0 & $P_{10}$ & 0.0523 \\
\hline & 0 & 0 & 0.0034 & 0 & 0.0009 & 0.0544 & -0.0544 & 0.0023 & 0 & 0 & $P_{9}$ & 0.0516 \\
\hline & 0 & 0 & 0.0035 & 0.0063 & 0.0009 & 0.0566 & 0.0564 & 0.0023 & 0 & 0 & $P_{8}$ & 0.0547 \\
\hline
\end{tabular}

Table 5. Fixed points and eigenvalues for vector-like fermions in the fundamental representation of $\mathrm{SU}_{c}(3)$, in the 210 approximation scheme, with $N_{f}=1$. We highlight in green the fixed points that appear also in the 321 approximation scheme. The labels in the second to the last last column refer to the list in table 12. The last column gives the values of the ratio $\rho$ for $\alpha_{2}$ or $\alpha_{3}$ depending on the case (see 2.15).

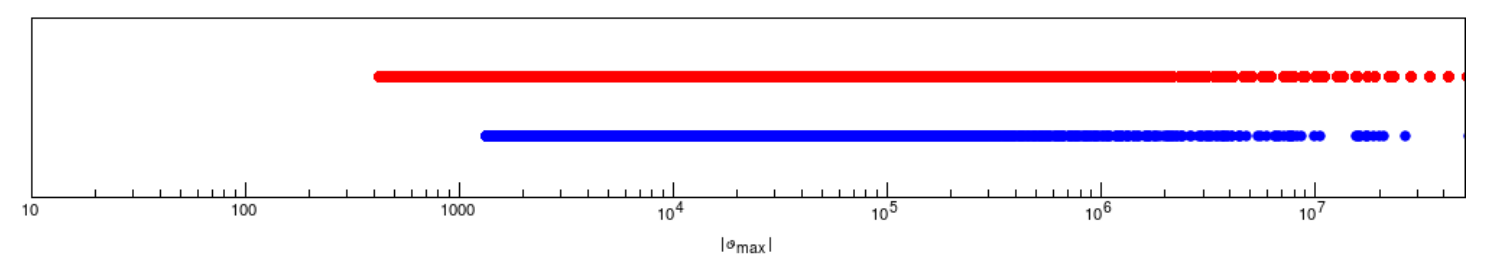

Figure 6. Distribution of the largest eigenvalue $\vartheta_{\max }$ of the stability matrix of the fixed points of the $\mathrm{SU}(3)$ adjoint representation. Blue: eigenvalues for the $Y$-independent solutions. Red: eigenvalues for the $Y$-dependent solutions. In both cases, there is no gap and the eigenvalues start at very large values. 


\begin{tabular}{|c|c|c|c|c|c|c|c|c|c|c|c|c|}
\hline$\left(N_{f}, l\right)$ & $\alpha_{1}^{*}$ & $\alpha_{2}^{*}$ & $\alpha_{3}^{*}$ & $\alpha_{t}^{*}$ & $\alpha_{y}^{*}$ & $\vartheta_{1}$ & $\vartheta_{2}$ & $\vartheta_{3}$ & $\vartheta_{4}$ & $\vartheta_{5}$ & & $\rho$ \\
\hline \multirow[t]{4}{*}{$\left(2, \frac{1}{2}\right)$} & 0 & 0 & 0.176 & 0 & 0 & -2.81 & -2.81 & 1.52 & 0 & 0 & $P_{11}$ & 5.45 \\
\hline & 0 & 0 & 0.205 & 0.365 & 0 & 3.84 & -3.28 & 1.52 & 0 & 0 & $P_{10}$ & 7.21 \\
\hline & 0 & 0 & 0.260 & 0 & 0.260 & 5.91 & -4.16 & 1.59 & 0 & 0 & $P_{9}$ & 11.1 \\
\hline & 0 & 0 & 0.330 & 0.588 & 0.330 & 8.99 & 5.29 & 1.68 & 0 & 0 & $P_{8}$ & 17.4 \\
\hline \multirow[t]{4}{*}{$(2,1)$} & 0 & 0 & 0.0600 & 0 & 0 & -0.960 & -0.960 & 0.360 & 0 & 0 & $P_{11}$ & 1.27 \\
\hline & 0 & 0 & 0.0646 & 0.115 & 0 & 1.08 & -1.03 & 0.371 & 0 & 0 & $P_{10}$ & 1.44 \\
\hline & 0 & 0 & 0.0727 & 0 & 0.0529 & 1.30 & -1.16 & 0.390 & 0 & 0 & $P_{9}$ & 1.77 \\
\hline & 0 & 0 & 0.0795 & 0.141 & 0.0578 & 1.50 & 1.27 & 0.405 & 0 & 0 & $P_{8}$ & 2.07 \\
\hline \multirow{4}{*}{$\left(2, \frac{3}{2}\right)$} & 0 & 0 & 0.0221 & 0 & 0 & -0.354 & -0.354 & 0.0737 & 0 & 0 & $P_{11}$ & 0.384 \\
\hline & 0 & 0 & 0.0232 & 0.0413 & 0 & 0.376 & -0.371 & 0.0764 & 0 & 0 & $P_{10}$ & 0.415 \\
\hline & 0 & 0 & 0.0252 & 0 & 0.0144 & 0.417 & -0.403 & 0.0810 & 0 & 0 & $P_{9}$ & 0.475 \\
\hline & 0 & 0 & 0.0266 & 0.0473 & 0.0152 & 0.448 & 0.426 & 0.0842 & 0 & 0 & $P_{8}$ & 0.520 \\
\hline \multirow[t]{4}{*}{$(2,2)$} & 0 & 0 & 0.0033 & 0 & 0 & -0.0530 & -0.0530 & 0.0022 & 0 & 0 & $P_{11}$ & 0.0495 \\
\hline & 0 & 0 & 0.0034 & 0.0061 & 0 & 0.0550 & -0.0549 & 0.0023 & 0 & 0 & $P_{10}$ & 0.0523 \\
\hline & 0 & 0 & 0.0036 & 0 & 0.0017 & 0.0587 & -0.0584 & 0.0024 & 0 & 0 & $P_{9}$ & 0.0579 \\
\hline & 0 & 0 & 0.0038 & 0.0068 & 0.0018 & 0.0612 & 0.0608 & 0.0025 & 0 & 0 & $P_{8}$ & 0.0616 \\
\hline \multirow{4}{*}{$\left(3, \frac{1}{2}\right)$} & 0 & 0 & 0.0600 & 0 & 0 & -0.960 & -0.960 & 0.360 & 0 & 0 & $P_{11}$ & 1.27 \\
\hline & 0 & 0 & 0.0646 & 0.115 & 0 & 1.08 & -1.03 & 0.371 & 0 & 0 & $P_{10}$ & 1.44 \\
\hline & 0 & 0 & 0.0882 & 0 & 0.0784 & 1.77 & -1.41 & 0.423 & 0 & 0 & $P_{9}$ & 2.47 \\
\hline & 0 & 0 & 0.0985 & 0.175 & 0.0876 & 2.10 & 1.58 & 0.443 & 0 & 0 & $P_{8}$ & 3.01 \\
\hline \multirow[t]{4}{*}{$(3,1)$} & 0 & 0 & 0.0114 & 0 & 0 & -0.182 & -0.182 & 0.0227 & 0 & 0 & $P_{11}$ & 0.182 \\
\hline & 0 & 0 & 0.0118 & 0.0210 & 0 & 0.191 & -0.189 & 0.0235 & 0 & 0 & $P_{10}$ & 0.195 \\
\hline & 0 & 0 & 0.0143 & 0 & 0.0095 & 0.237 & -0.229 & 0.0276 & 0 & 0 & $P_{9}$ & 0.264 \\
\hline & 0 & 0 & 0.0150 & 0.0267 & 0.0100 & 0.252 & 0.241 & 0.0288 & 0 & 0 & $P_{8}$ & 0.288 \\
\hline \multirow[t]{4}{*}{$\left(4, \frac{1}{2}\right)$} & 0 & 0 & 0.0221 & 0 & 0 & -0.354 & -0.354 & 0.0737 & 0 & 0 & $P_{11}$ & 0.384 \\
\hline & 0 & 0 & 0.0232 & 0.0413 & 0 & 0.376 & -0.371 & 0.0764 & 0 & 0 & $P_{10}$ & 0.415 \\
\hline & 0 & 0 & 0.0335 & 0 & 0.0268 & 0.607 & -0536 & 0.0987 & 0 & 0 & $P_{9}$ & 0.763 \\
\hline & 0 & 0 & 0.0361 & 0.0642 & 0.0289 & 0.670 & 0.577 & 0.104 & 0 & 0 & $P_{8}$ & 0.866 \\
\hline \multirow[t]{4}{*}{$\left(5, \frac{1}{2}\right)$} & 0 & 0 & 0.0033 & 0 & 0 & -0.0530 & -0.530 & 0.0022 & 0 & 0 & $P_{11}$ & 0.0495 \\
\hline & 0 & 0 & 0.0343 & 0.0061 & 0 & 0.0550 & -0.0549 & 0.0023 & 0 & 0 & $P_{10}$ & 0.0523 \\
\hline & 0 & 0 & 0.0052 & 0 & 0.0038 & 0.0850 & -0.0829 & 0.0034 & 0 & 0 & $P_{9}$ & 0.1010 \\
\hline & 0 & 0 & 0.0055 & 0.0097 & 0.0040 & 0.0903 & 0.0878 & 0.035 & 0 & 0 & $P_{8}$ & 0.111 \\
\hline
\end{tabular}

Table 6. Same as table 5 but with $N_{f}>1$.

fixed points have $\delta a$ of $O(100)$. Tests of the $c$-function confirm these results, even though the $a$-function seems to be more sensitive, in the sense that it suffers greater relative change.

Again, we come up empty handed. The models with the vector-like fermions in the adjoint representation of $\mathrm{SU}_{c}(3)$ do not provide a viable AS extension to the SM.

Higher $\mathrm{SU}_{c}(3)$ representations are disfavored by experimental constraints because of the early onset of the modifications in the $\alpha_{3}$ running.

\subsubsection{A model that almost works}

Having ruled out all possible candidates, one may wonder if the criteria in (2.3) and (2.9) might be too stringent and make us miss some potentially interesting models. In the case at hand, we can indeed find additional fixed points that naively seem to be good candidates for an asymptotically safe extension of the SM. This is achieved if we allow for larger values of $\vartheta$ and relinquish the condition (2.9).

As an example, consider the case with the vector-like fermions in the representations with $N_{f}=3, \ell=1 / 2$ and $Y=3 / 2$. Its fixed points and eigenvalues are given in table 8 .

This example provides a very interesting (and non-trivial) extension of the SM which includes non-trivial fixed point value for the gauge coupling $\alpha_{1}$ as well as the Yukawa 


\begin{tabular}{|c|c|c|c|c|c|c|c|c|c|c|c|c|c|c|}
\hline$\left(N_{f}, l\right)$ & $\alpha_{1}^{*}$ & $\alpha_{2}^{*}$ & $\alpha_{3}^{*}$ & $\alpha_{t}^{*}$ & $\alpha_{y}^{*}$ & $\alpha_{\lambda}^{*}$ & $\vartheta_{1}$ & $\vartheta_{2}$ & $\vartheta_{3}$ & $\vartheta_{4}$ & $\vartheta_{5}$ & $\vartheta_{6}$ & $\sigma$ & $\rho$ \\
\hline \multirow[t]{2}{*}{$\left(1, \frac{1}{2}\right)$} & 0 & 0.0291 & 0 & 0.0148 & 0 & 0.0120 & -0.306 & 0.208 & 0.132 & 0.0827 & 0 & 0 & 0.737 & 0.263 \\
\hline & 0 & 0.0305 & 0 & 0.0155 & 0.0209 & 0.0126 & 0.322 & 0.219 & 0.139 & 0.0863 & 0 & 0 & 0.719 & 0.281 \\
\hline \multirow[t]{2}{*}{$\left(1, \frac{5}{2}\right)$} & 0 & 0 & 0.0346 & 0 & 0 & 0 & -0.748 & -0.748 & 0.295 & 0 & 0 & 0 & 0.577 & 0.423 \\
\hline & 0 & 0 & 0.0355 & 0 & 0.0167 & 0 & -0.774 & 0.768 & 0.304 & 0 & 0 & 0 & 0.559 & 0.441 \\
\hline \multirow[t]{2}{*}{$(1,3)$} & 0 & 0 & 0.0252 & 0 & 0 & 0 & -0.501 & -0.501 & 0.156 & 0 & 0 & 0 & 0.676 & 0.323 \\
\hline & 0 & 0 & 0.0258 & 0 & 0.0101 & 0 & -0.516 & 0.514 & 0.160 & 0 & 0 & 0 & 0.664 & 0.336 \\
\hline \multirow[t]{4}{*}{$\left(1, \frac{7}{2}\right)$} & 0 & 0 & 0.0171 & 0 & 0 & 0 & -0.315 & -0.315 & 0.0670 & 0 & 0 & 0 & 0.771 & 0.228 \\
\hline & 0 & 0 & 0.0177 & 0.0358 & 0 & 0.0221 & 0.969 & -0.329 & 0.290 & 0.0723 & 0 & 0 & 0.758 & 0.242 \\
\hline & 0 & 0 & 0.0175 & 0 & 0.0058 & 0 & -0.324 & 0.324 & 0.0717 & 0 & 0 & 0 & 0.763 & 0.237 \\
\hline & 0 & 0 & 0.0182 & 0.0368 & 0.0061 & 0.0227 & 0.998 & 0.334 & 0.298 & 0.0742 & 0 & 0 & 0.748 & 0.252 \\
\hline \multirow[t]{4}{*}{$(1,4)$} & 0 & 0 & 0.098 & 0 & 0 & 0 & -0.170 & -0.170 & 0.0223 & 0 & 0 & 0 & 0.864 & 0.136 \\
\hline & 0 & 0 & 0.0102 & 0.0193 & 0 & 0.0119 & 0.521 & -0.177 & 0.165 & 0.0231 & 0 & 0 & 0.856 & 0.144 \\
\hline & 0 & 0 & 0.0101 & 0 & 0.0029 & 0 & -0.175 & 0.175 & 0.0229 & 0 & 0 & 0 & 0.859 & 0.141 \\
\hline & 0 & 0 & 0.0104 & 0.0198 & 0.0030 & 0.0123 & 0.536 & 0.182 & 0.170 & 0.0237 & 0 & 0 & 0.8505 & 0.149 \\
\hline \multirow[t]{4}{*}{$\left(1, \frac{9}{2}\right)$} & 0 & 0 & 0.0032 & 0 & 0 & 0 & -0.0519 & -0.0519 & 0.0022 & 0 & 0 & 0 & 0.955 & 0.0451 \\
\hline & 0 & 0 & 0.0033 & 0.0059 & 0 & 0.0037 & 0.159 & -0.0537 & 0.0526 & 0.0023 & 0 & 0 & 0.952 & 0.0476 \\
\hline & 0 & 0 & 0.0032 & 0 & 0.0008 & 0 & -0.0532 & 0.0532 & 0.0023 & 0 & 0 & 0 & 0.953 & 0.0469 \\
\hline & 0 & 0 & 0.0033 & 0.0061 & 0.0009 & 0.00038 & 0.1635 & 0.0551 & 0.0540 & 0.0023 & 0 & 0 & 0.9505 & 0.0495 \\
\hline \multirow[t]{2}{*}{$(2,1)$} & 0 & 0 & 0.346 & 0 & 0 & 0 & -0.748 & -0.748 & 0.295 & 0 & 0 & 0 & 0.577 & 0.423 \\
\hline & 0 & 0 & 0.0381 & 0 & 0.0319 & 0 & -0.846 & 0.824 & 0.326 & 0 & 0 & 0 & 0.5077 & 0.492 \\
\hline \multirow[t]{3}{*}{$\left(2, \frac{3}{2}\right)$} & 0 & 0 & 0.0171 & 0 & 0 & 0 & -0.315 & -0.315 & 0.0699 & 0 & 0 & 0 & 0.771 & 0.228 \\
\hline & 0 & 0 & 0.0177 & 0.0358 & 0 & 0.0221 & 0.969 & -0.329 & 0.295 & 0.0723 & 0 & 0 & 0.758 & 0.242 \\
\hline & 0 & 0 & 0.0187 & 0 & 0.0113 & 0 & -0.350 & 0.349 & 0.0767 & 0 & 0 & 0 & 0.737 & 0.263 \\
\hline \multirow[t]{4}{*}{$(2,2)$} & 0 & 0 & 0.0032 & 0 & 0 & 0 & -0.0519 & -0.0519 & 0.0022 & 0 & 0 & 0 & 0.955 & 0.0451 \\
\hline & 0 & 0 & 0.0033 & 0.0059 & 0 & 0.0037 & 0.159 & -0.0537 & 0.0526 & 0.0023 & 0 & 0 & 0.952 & 0.0476 \\
\hline & 0 & 0 & 0.0035 & 0 & 0.0016 & 0 & -0.0570 & 0.0570 & 0.0024 & 0 & 0 & 0 & 0.948 & 0.0521 \\
\hline & 0 & 0 & 0.0036 & 0.0065 & 0.0017 & 0.0040 & 0.1756 & 0.0592 & 0.0579 & 0.0025 & 0 & 0 & 0.945 & 0.552 \\
\hline \multirow[t]{2}{*}{$\left(3, \frac{1}{2}\right)$} & 0 & 0 & 0.0346 & 0 & 0 & 0 & -0.748 & -0.748 & 0.295 & 0 & 0 & 0 & 0.577 & 0.423 \\
\hline & 0 & 0 & 0.0417 & 0 & 0.0440 & 0 & -0.950 & 0.913 & 0.359 & 0 & 0 & 0 & 0.431 & 0.569 \\
\hline \multirow[t]{4}{*}{$(3,1)$} & 0 & 0 & 0.0098 & 0 & 0 & 0 & -0.170 & -0.170 & 0.0223 & 0 & 0 & 0 & 0.864 & 0.136 \\
\hline & 0 & 0 & 0.0102 & 0.0193 & 0 & 0.119 & 0.521 & -0.177 & 0.165 & 0.0231 & 0 & 0 & 0.856 & 0.144 \\
\hline & 0 & 0 & 0.0118 & 0 & 0.0081 & 0 & 0.208 & -0.208 & 0.0270 & 0 & 0 & 0 & 0.819 & 0.181 \\
\hline & 0 & 0 & 0.0123 & 0.0237 & 0.0085 & 0.0147 & 0.641 & 0.218 & 0.200 & 0.0281 & 0 & 0 & 0.8062 & 0.194 \\
\hline \multirow[t]{3}{*}{$\left(4, \frac{1}{2}\right)$} & 0 & 0 & 0.0171 & 0 & 0 & 0 & -0.315 & -0.315 & 0.0699 & 0 & 0 & 0 & 0.771 & 0.228 \\
\hline & 0 & 0 & 0.0177 & 0.0358 & 0 & 0.0221 & 0.969 & -0.329 & 0.290 & 0.0723 & 0 & 0 & 0.758 & 0.242 \\
\hline & 0 & 0 & 0.0226 & 0 & 0.0196 & 0 & 0.439 & -0.437 & 0.0931 & 0 & 0 & 0 & 0.647 & 0.353 \\
\hline \multirow[t]{4}{*}{$\left(5, \frac{1}{2}\right)$} & 0 & 0 & 0.0033 & 0 & 0 & 0 & -0.0519 & -0.0519 & 0.0022 & 0 & 0 & 0 & 0.955 & 0.0451 \\
\hline & 0 & 0 & 0.0033 & 0.0059 & 0 & 0.0037 & 0.159 & -0.0537 & 0.0526 & 0.0023 & 0 & 0 & 0.952 & 0.0476 \\
\hline & 0 & 0 & 0.0048 & 0 & 0.0035 & 0 & 0.0798 & -0.0793 & 0.0034 & 0 & 0 & 0 & 0.914 & 0.0859 \\
\hline & 0 & 0 & 0.0050 & 0.0092 & 0.0037 & 0.0057 & 0.248 & 0.0843 & 0.0809 & 0.0035 & 0 & 0 & 0.9066 & 0.0934 \\
\hline
\end{tabular}

Table 7. Fixed points and eigenvalues for vector-like fermions in the fundamental representation of $\mathrm{SU}_{c}(3)$, in the 321 approximation scheme. The last two columns give the values of the ratio $\sigma$ and $\rho$ for $\alpha_{2}$ or $\alpha_{3}$ depending on the case (see 2.15).

\begin{tabular}{|c|c|c|c|c|c|c|c|c|c|c|c|}
\hline$\left(N_{f}, \ell, Y\right)$ & $\alpha_{1}^{*}$ & $\alpha_{2}^{*}$ & $\alpha_{3}^{*}$ & $\alpha_{t}^{*}$ & $\alpha_{y}^{*}$ & $\vartheta_{1}$ & $\vartheta_{2}$ & $\vartheta_{3}$ & $\vartheta_{4}$ & $\vartheta_{5}$ & $\rho_{1}$ \\
\hline$(3,1 / 2,3 / 2)$ & 0.188 & 0 & 0 & 0 & 0.778 & 33.2 & -3.36 & -0.817 & 0 & 0 & 2.69 \\
\hline
\end{tabular}

Table 8. Values of the couplings at the fixed point, eigenvalues and $\rho_{1}$ ratio for the model that almost works (210 approximation scheme). 


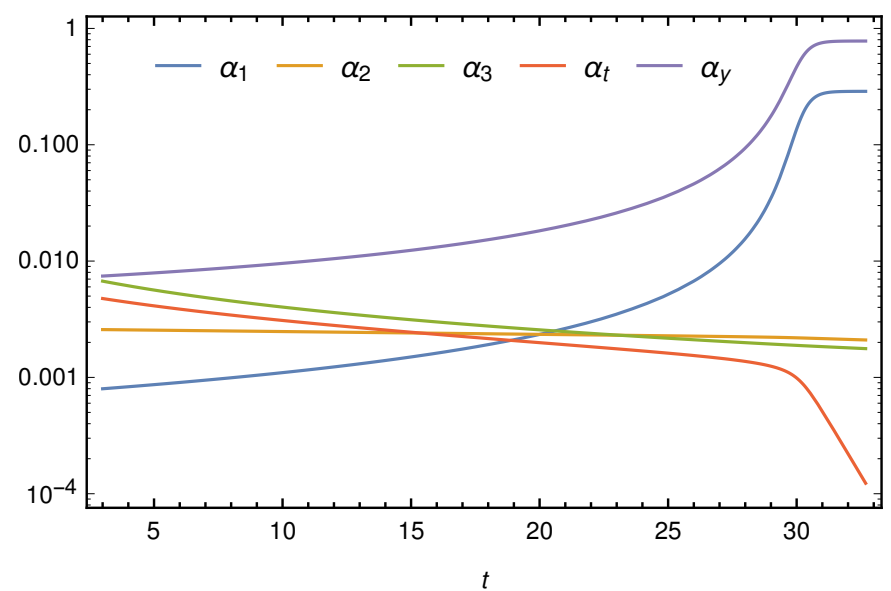

Figure 7. Evolution of the couplings with $t$ in a logarithmic scale for the fixed point in table 8). This running provides a trajectory in the theory space connecting the fixed point and the physics at a matching scale around $2 \mathrm{TeV}$.

coupling $\alpha_{y}$ (remember that the quartic scalar interaction in the 210 scheme does not renormalize).

We see that some of the scaling exponents $\vartheta_{i}$ are large and the criterion (2.9) is accordingly violated. Nonetheless, let us momentarily suspend disbelief and apply the formula in (2.3). We do not find any coupling frozen to zero and therefore a SM matching seems plausible.

In fact, taking $M=m_{Z} \exp (3) \simeq 1.83 \mathrm{TeV}$ - where the SM couplings have the values $\alpha_{1}=0.000795, \alpha_{2}=0.00257, \alpha_{3}=0.00673, \alpha_{t}=0.00478$ - we find a good matching, with an error of the order of per mille (see figure 7 ).

But for the large scaling exponents, this model seems to provide a very promising candidate for an AS extension of the SM. Yet it is not radiatively stable - a fact that vindicates the role of criteria in (2.9) as a filter for the physical fixed points. The 321 approximation scheme $\beta$-functions generate very different fixed points that cannot be easily traced back to those in the 210 approximation scheme. Moreover, all these fixed points have a trivial coupling and cannot provide a viable extension to the SM.

\subsubsection{Five benchmark models studied in the literature}

The authors of [15] find that it is possible to generate asymptotically safe extensions to the $\mathrm{SM}$ in the subsystem $\left(\alpha_{2}, \alpha_{3}, \alpha_{y}\right)$ of the couplings. The five benchmark models discussed in [15] (labelled as A, B, C, D and E) are not among those in our scan because they do not include hypercharge, top Yukawa and quartic interaction. We analysed them separately.

The hypercharge $Y$ can easily be added to these models. The charge $Y$ must be larger than a minimal value in order for the corresponding direction in the UV critical surface to be marginally relevant. This does not change the behavior of the models.

Similar to what happens to the model in section 4.2.4, all these models have at least one of their scaling exponents rather large (See table 9). The large values of $\vartheta$ imply that the fixed points are not in the perturbative domain even though they can be connected to 


\begin{tabular}{|c|c|c|c|c|c|c|c|c|}
\hline & $\left(R_{3}, R_{2}, N_{f}\right)$ & $\alpha_{2}^{*}$ & $\alpha_{3}^{*}$ & $\alpha_{y}^{*}$ & $\vartheta_{1}$ & $\vartheta_{2}$ & $\vartheta_{3}$ & $\rho$ \\
\hline$A$ & $(1,4,12)$ & 0.241 & 0 & 0.338 & 210 & -1.90 & 0 & 45.3 \\
\hline$B$ & $(10,1,30)$ & 0 & 0.129 & 0.116 & 338 & -2.06 & 0 & 107 \\
& & 0.277 & 0.129 & 0.116 & 341 & -2.08 & 0.897 & 107 \\
\hline$C$ & $(10,4,80)$ & 0 & 0.332 & 0.0995 & 23258 & -2.18 & 0 & 9138 \\
& & 0.0753 & 0.0503 & 0.0292 & 1499 & 328 & -2.77 & 630 \\
& & 0.800 & 0 & 0.150 & 145193 & -2.12 & 0 & 57378 \\
\hline$D$ & $(3,4,290)$ & 0.0615 & 0.0416 & 0.0057 & 943 & 45.3 & -2.29 & 371 \\
& & 0.0896 & 0 & 0.0067 & 1984 & -2.11 & 0 & 781 \\
\hline$E$ & $(3,3,72)$ & 0.218 & 0.150 & 0.0471 & 896 & 112 & -1.78 & 326 \\
\hline
\end{tabular}

Table 9. Couplings, eigenvalues and the ratios $\rho_{i}$, with $i=2,3$ depending on the case, for the benchmark models in [15] for the 210 approximation scheme.

\begin{tabular}{|c|c|c|c|c|c|c|c|c|}
\hline & $\left(R_{3}, R_{2}, N_{f}\right)$ & $\alpha_{2}^{*}$ & $\alpha_{3}^{*}$ & $\alpha_{y}^{*}$ & $\vartheta_{1}$ & $\vartheta_{2}$ & $\vartheta_{3}$ & $\rho_{3}$ \\
\hline$A$ & $(1,4,12)$ & 0 & 0 & 0.1509 & -4.83 & 0 & 0 & - \\
\hline$B$ & $(10,1,30)$ & 0 & 0.0138 & 0 & -20.02 & 2.24 & 0 & 3.14 \\
& & 0 & 0 & 0.0594 & -4.75 & 0 & 0 & - \\
\hline$C$ & $(10,4,80)$ & 0 & 0 & 0.0187 & -4.501 & 0 & 0 & - \\
& & 0 & 0.0036 & 0 & -49.4 & 2.28 & 0 & 9.29 \\
\hline$D$ & $(3,4,290)$ & 0 & 0 & 0.0115 & -6.95 & 0 & 0 & - \\
& & 0 & 0.0108 & 0 & -36.7 & 1.015 & 0 & 5.81 \\
\hline$E$ & $(3,3,72)$ & 0 & 0 & 0.0357 & -5.79 & 0 & 0 & - \\
& & 0 & 0.0305 & 0 & -21.8 & 1.098 & 0 & 2.66 \\
\hline
\end{tabular}

Table 10. Couplings, eigenvalues and the ratio $\rho_{3}$ for the benchmark models in [15] for the 321 approximation scheme.

the SM in the IR regime. The fixed points in the 210 approximation scheme cannot be connected to those in the 321 approximation scheme because of their instability against radiative corrections. We can see how the structure of the fixed points changes by comparing table 9 to table 10. The eigenvalues are always large in both tables.

If we take the fixed points in the 321 approximation scheme at their face value and try to match them to the SM, we always encounter a coupling, $\alpha_{2}$ in almost all the cases (see table 10), that is frozen to its vanishing value: the theory is trivial in the coupling $\alpha_{2}$ and it cannot be matched to the SM. In other words, the benchmark models in [15] suffer from the same pathology as the models in our scan. Unlike those models, in this case it is a non-abelian coupling that is trivial. This is worrisome and should be born in mind if one were to entertain the idea of embedding $U_{Y}(1)$ in a non-abelian group in order to find an AS extension of the SM. 


\begin{tabular}{|c|c|c|c|c|c|c|c|c|c|c|c|c|c|}
\hline$\alpha_{1}^{*}$ & $\alpha_{2}^{*}$ & $\alpha_{3}^{*}$ & $\alpha_{t}^{*}$ & $\alpha_{y}^{*}$ & $\alpha_{z}^{*}$ & $\vartheta_{1}$ & $\vartheta_{2}$ & $\vartheta_{3}$ & $\vartheta_{4}$ & $\vartheta_{5}$ & $\vartheta_{6}$ & $\rho_{2}$ & $\rho_{1}$ \\
\hline 0.226 & 0.193 & 0 & 0 & 0.778 & 0.534 & 241 & 24.2 & -2.85 & -2.28 & -1.51 & 0 & 0 & 0 \\
\hline
\end{tabular}

Table 11. Values of the couplings at the fixed point of interest, eigenvalues and $\rho$ ratios for the model combining 3 fields in the representation $(1,2,3 / 2)$ and 8 fields in the representation $(1,5,0)$ (210 approximation scheme).

\subsubsection{Two more benchmark models studied in the literature}

The authors of [16] study three models where the fermions are in the representations $\left(N_{f}, \ell, Y\right)=(3,2,1 / 6),(3,1,0) \oplus(1,2,1 / 2)$ and $(3,1,0) \oplus(1,3,0) \oplus(1,1,1)$, respectively. Further models (with Majorana fermions charged under only one gauge group at the time) are introduced in [17]. In both papers, they consider the large $N_{f}$ limit and the zeros of the $\beta$-functions are found after resumming the blob diagrams of the perturbative theory.

Resummed $\beta$-functions could help in discussing the AS of a theory, yet there is no available procedure that is free of the ambiguities deriving from summing over a particular class of diagrams. Moreover, a large number of new states must be included to be within the regime of validity of the resummation scheme which — from the phenomenological point of view - is unappealing.

It is difficult to compare these results to ours because of the non-perturbative nature of the resummation procedure. In our approach these models are all ruled out because of the larger scaling dimensions we expect for the given large values of $N_{f}$ (see figure 4 and the discussion in section 4). The fixed points obtained in the approach of [16] and [17] are probably linked to essential singularities in the complete resummation [58] and no perturbative treatment - like expanding around the fixed point values to search for the trajectory back to the SM - is possible.

While these models provide interesting examples of AS theories, it is difficult to see them as viable candidate for extensions of the SM because the low-energy matching is problematic: to wit, even assuming that the fixed point thus found are physical, the authors of [17] conclude (in the published version of their paper) that there is no matching because of the persistence of the Landau pole in the U(1) coupling (which can only be avoided at the price of making the vacuum of the model unstable because of the running of the quartic Higgs interaction).

\subsubsection{Combining more than one representation}

Combining vector-like fermions in different representations (as done, for instance, in [16, 17]) provides other examples of models that almost work. In the simplest scenario, we can try to construct a model with two types of vector-like fermions. In that case, we duplicate the last three terms in eq. (4.1) for fermions $\tilde{\psi}$ and scalars $\tilde{S}$. We call the extra Yukawa coupling $z$ with, as usual,

$$
\alpha_{z}=\frac{z^{2}}{(4 \pi)^{2}}
$$

and assume no mixing between the two families. 


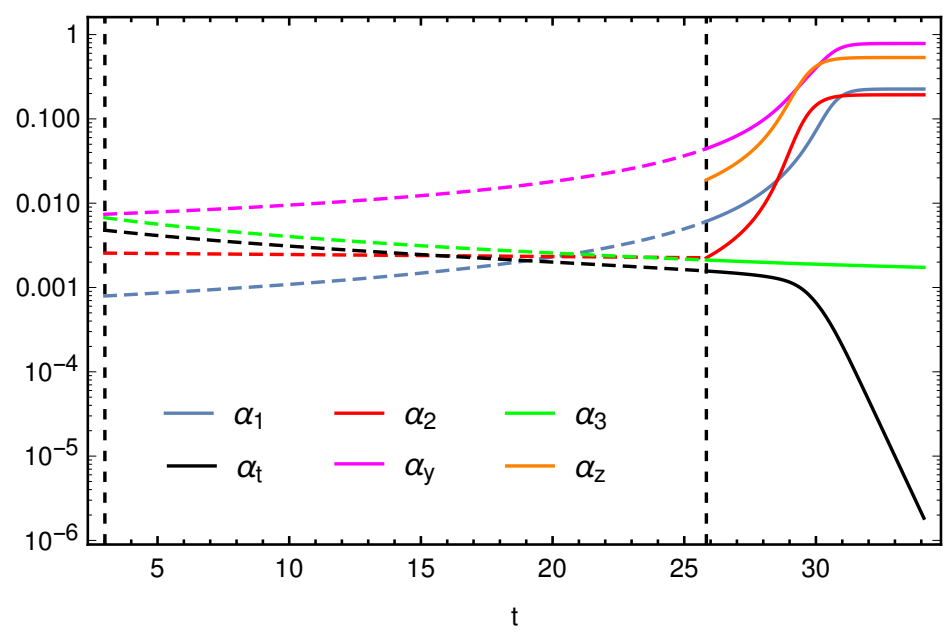

Figure 8. Evolution of the couplings with $t$ for the fixed point in table 11 within the 210 approximation with 3 fields in $(1,2,3 / 2)$ and 8 fields in $(1,5,0)$. This running provides a trajectory in the theory space connecting the fixed point to a matching scale around $2 \mathrm{TeV}$ passing through another matching (for the quintuplets) at about $10^{13} \mathrm{TeV}$.

Since many of the BSM extensions attempt to describe dark matter, we take one of the possible minimal models discussed in [59] and identify some of the vector-like fermions with dark matter. This exercise makes clear the potential relevance of AS in selecting physics BSM.

We take $N_{f_{2}}$ vector-like fermions with quantum numbers $p=q=0, \ell=2$ and $Y=0$. That is, we take colorless quintuplets with no hypercharge. Within the 210 approximation scheme, for the combination $(1,2,3 / 2) \oplus(1,5,0)$, we realize that fixed points split in two categories: fixed points that depend on the number of quintuplets $N_{f_{2}}$ and fixed points that do not. Clearly, the latter have $\alpha_{y}=0$ so that the vector-like fermions enter only via loops in the gauge $\beta$-functions. Consequently, the conditions to lie on the critical surface of those fixed points imply that $\alpha_{2}=0$. This feature makes the corresponding fixed points uninteresting.

For the remaining fixed points, we find that in order to have $\alpha_{i}<1$ for all couplings, the minimum number of quintuplets should be equal to eight. Taking the minimal case of $N_{f_{2}}=8$, we find 6 fixed points, all of them having one large eigenvalue around 250. Thus, according to our requirement about perturbation theory, these fixed points are not reliable since there is always one $\vartheta$ which is much larger than 1 . This is similar to what happens in section 4.2.4.

Nevertheless, we can find a matching with the SM. The only difference with respect to the model in section 4.2.4 is that, in the present case, two matching scales are needed - the reason being that the large number of quintuplets makes $\alpha_{2}$ decrease fast so that these fields must be decoupled at very high energies. In figure 8 we show the logarithmic running of the couplings and the two different matching scales. The quintuplets decouple at an energy scale $O\left(10^{13}\right) \mathrm{TeV}$ (and must be considered wimpzilla dark matter [60]), the doublets at the energy scale of $1.83 \mathrm{TeV}$. All the couplings flow to the fixed point in table 11 
Even though figure 8 shows a nice flow of the coupling constants toward the SM, the size of the eigenvalues spells doom and the likely breakdown of perturbation theory. Indeed, the fixed point analysed does not survive in the 321 approximation scheme and the model does not work.

\section{Conclusions}

A systematic scan (covering 378,000 models) of possible extensions of the SM based on vector-like fermions charged under the SM groups and carrying various representations and coming in several copies (generations) shows that there are no fixed points in the $\beta$ function of the models that satisfy the minimal criteria to make them physically acceptable. Most of those that appear in the 210 approximation scheme are difficult to identify when probed in the 321 approximation scheme, and therefore are almost certainly artifacts of the approximation. Those that seem to be present in both schemes (or appear only at the higher order) always contain a trivial solution in which at least one of the couplings is frozen to zero thus suggesting that the Landau problem of the LO theory persists at higher orders in the perturbative expansion.

We conclude that it is not possible, at least with the 378,000 models we have examined, to extend the SM up to arbitrarily high energies in perturbation theory. The same happens with the models discussed in section 4.2.4 and 4.2.7, and the five models proposed as benchmarks in [15]: the 321 approximation scheme $\beta$-functions generate very different fixed points that cannot be easily traced back to those in the 210 approximation scheme, and all the fixed points in 321 approximation scheme have at least one trivial coupling.

Our search for AS extensions of the SM has returned a negative answer and no viable candidate. Whenever perturbation theory may reasonably be assumed to give reliable answers, the issue is triviality of some gauge coupling: usually the abelian one, but sometimes also a non-abelian one.

There are various possible ways out of this situation. The most straightforward would be to keep the same gauge group but to consider models with BSM fields more complicated than vector-like fermions. Insofar as the vector-like fermions can be viewed as a proxy for more general matter fields, this does not seem too promising a line of enquiry. Another possibility is to embed the SM gauge group in a larger grand unification group before AS becomes manifest. This is actually the standard answer to the $\mathrm{U}(1)$ triviality issue. Promising work along these lines is already under way [61]. We just remark here that having a non-abelian group does not by itself guarantee the absence of the triviality issue, as some of our examples show.

All in all, the most plausible scenario seems that in which the fixed point making the SM AS, if it exists at all, lies outside the perturbative regime and accordingly is inherently invisible to our approach. An example is provided by non-perturbative computations based on a large- $N_{f}$ approximation [17].

A completely different possibility is that the Landau pole is cured by gravity. Gravity gives contributions to the $\beta$-functions that are linear in Newton's coupling and in the matter couplings, and hence generate non-trivial fixed points for the latter. Some of these fixed 
points, possessing few relevant directions, greatly constrain the allowed RG trajectories and lead to predictions for low energy couplings. For the abelian gauge coupling this has been discussed in [62, 63]. For related results see also [64, 65].

\section{Acknowledgments}

We are very grateful to Luminita Mihaila for giving us access to her results on the 3-loop $\beta$-functions for the gauge couplings in non-simple groups prior to their publication. We also thank Daniel Litim and Francesco Sannino for useful discussions.

\section{A Analysis of marginal couplings}

Here we prove the statement, made in section 2.3, that when the marginal couplings are associated to vanishing gauge couplings, the behavior of the flow at quadratic order is determined by the coefficients $P_{i i i}$.

The general form of the gauge $\beta$-functions is

$$
\beta_{i}=\left(A^{i}+B_{r}^{i} \alpha_{r}+C_{r s}^{i} \alpha_{r} \alpha_{s}\right) \alpha_{i}^{2},
$$

where $A^{i}, B_{r}^{i}$ and $C_{r s}^{i}$ represent the one, two and three-loops coefficients. Their contribution to the stability matrix is given by

$$
M_{i j}=\left.\frac{\partial \beta_{i}}{\partial \alpha_{j}}\right|_{\alpha_{i}^{*}}=\left(B_{j}^{i}+2 C_{j r}^{i} \alpha_{r}^{*}\right) \alpha_{i}^{* 2}+2\left(A^{i}+B_{r}^{i} \alpha_{r}^{*}+C_{r s}^{i} \alpha_{r}^{*} \alpha_{s}^{*}\right) \alpha_{i}^{*} \delta_{i j} .
$$

We see that if $\alpha_{i}^{*}=0$, the row $i$ will have zeros in all the entries. This does not happen for the Yukawa interactions, whose NLO $\beta$-functions have the form $\beta_{Y_{i}}=\left(D_{r}^{i} \alpha_{r}+F_{r s}^{i} \alpha_{r} \alpha_{s}\right) \alpha_{i}$. Then, the contribution to the stability matrix reads

$$
M_{i j}=\left.\frac{\partial \beta_{Y_{i}}}{\partial \alpha_{j}}\right|_{\alpha_{i}^{*}}=\left(D_{j}^{i}+2 F_{j r}^{i} \alpha_{r}^{*}\right) \alpha_{i}^{*}+\left(D_{r}^{i} \alpha_{r}^{*}+F_{r s}^{i} \alpha_{r}^{*} \alpha_{s}^{*}\right) \delta_{i j}
$$

where we see that if $\alpha_{i}^{*}=0$, the last piece will be in general different from zero. Consequently, we do not have a row of zeros. The fact of having rows of zeros implies that $\operatorname{det} M=0$. Thus, the matrix $M$ is singular and there exist vectors $\mathbf{x}$ such that $A \mathbf{x}=0 \mathbf{x}$. As a result, $M$ has the eigenvalue $\lambda=0$ with multiplicity given by the number of zero rows.

Suppose we have a fixed point with two gauge couplings equal to zero. Then the stability matrix will have two zero rows, that we can assume to be the last two. This implies that the $n-2$ eigenvectors corresponding to $\lambda_{i} \neq 0$ have the form $\mathbf{V}^{i}=\left[V_{1}^{i}, V_{2}^{i}, \ldots, V_{n-2}^{i}, 0,0\right]$. The eigenvectors for $\lambda=0$ lie in a 2-dimensional plane. There is a freedom in choosing these vectors, and we can take them to have the form $\mathbf{V}^{n-1}=\left[V_{1}^{n-1}, V_{2}^{n-1}, \ldots, V_{n-2}^{n-1}, V_{n-1}^{n-1}, 0\right]$, $\mathbf{V}^{n}=\left[V_{1}^{n}, V_{2}^{n}, \ldots, V_{n-2}^{n}, 0, V_{n}^{n}\right]$. Moreover, the entries $V_{n-1}^{n-1}, V_{n}^{n}$ can be taken to be positive without loss of generality. Thus, the transformation matrix constructed with the 
eigenvectors of $M$ takes the form

$$
S=\left[\begin{array}{cccccc}
V_{1}^{1} & V_{1}^{2} & \ldots & V_{1}^{n-2} & V_{1}^{n-1} & V_{1}^{n} \\
V_{2}^{1} & V_{2}^{2} & \ldots & V_{2}^{n-2} & V_{2}^{n-1} & V_{2}^{n} \\
\vdots & \vdots & \ddots & \vdots & \vdots & \vdots \\
V_{n-2}^{1} & V_{n-2}^{2} & \ldots & V_{n-2}^{n-2} & V_{n-2}^{n-1} & V_{n-2}^{n} \\
0 & 0 & \ldots & 0 & V_{n-1}^{n-1} & 0 \\
0 & 0 & \ldots & 0 & 0 & V_{n}^{n}
\end{array}\right]
$$

This implies that

$$
S^{-1}=\left[\begin{array}{cccccc}
a_{1,1} & a_{1,2} & \ldots & a_{1, n-2} & a_{1, n-1} & a_{1, n} \\
a_{2,1} & a_{2,2} & \ldots & a_{2, n-2} & a_{2, n-1} & a_{2, n} \\
\vdots & \vdots & \ddots & \vdots & \vdots & \vdots \\
a_{n-2,1} & a_{n-2,2} & \ldots & a_{n-2, n-2} & a_{n-2, n-1} & a_{n-2, n} \\
0 & 0 & \ldots & 0 & b & 0 \\
0 & 0 & \ldots & 0 & 0 & c
\end{array}\right]
$$

where we have labelled $a_{i, j}$ the non-zero entries and we have called $b=1 / V_{n-1}^{n-1}, c=1 / V_{n}^{n}$. Now, when we compute the form of the new variables $z_{i}=S_{i j}^{-1} y_{j}=S_{i j}^{-1}\left(\alpha_{j}-\alpha_{j}^{*}\right)$, we observe that two of the new coordinates are just proportional to the asymptotically free variables, namely $z_{n-1}=b \cdot y_{n-1}=b \cdot \alpha_{n-1}, z_{n}=c \cdot y_{n}=c \cdot \alpha_{n}$. This result has an important effect in the analysis. For the gauge $\beta$-functions,

$$
\begin{aligned}
P_{i j k}=\left.\frac{\partial^{2} \beta_{i}}{\partial \alpha_{j} \alpha_{k}}\right|_{\alpha_{i}^{*}}= & 2 C_{j k}^{i} \alpha_{i}^{* 2}+2\left(B_{j}^{i}+2 C_{j r}^{i} \alpha_{r}^{*}\right) \alpha_{i}^{*} \delta_{i k}+2\left(B_{k}^{i}+2 C_{k r}^{i} \alpha_{r}^{*}\right) \alpha_{i}^{*} \delta_{i j} \\
& +2\left(A^{i}+B_{r}^{i} \alpha_{r}^{*}+C_{r s}^{i} \alpha_{r}^{*} \alpha_{s}^{*}\right) \delta_{i j} \delta_{i k}
\end{aligned}
$$

which in the case of the AF couplings reduces to

$$
P_{i j k}=2\left(A^{i}+B_{r}^{i} \alpha_{r}^{*}+C_{r s}^{i} \alpha_{r}^{*} \alpha_{s}^{*}\right) \delta_{i j} \delta_{i k}
$$

We conclude that in order to know if a marginal coupling is relevant or irrelevant we need only check the sign of $P_{i i i}$. If $P_{i i i}<0$, the coupling is marginally relevant. If $P_{i i i}>0$, the coupling is marginally irrelevant.

\section{B Conformal field theory and central charges}

The CFT at a given fixed point is characterized by two local functions: $c$ and $a$. We refer to them collectively as central charges or CFT functions. They appear in the matrix element of the trace of the energy-momentum tensor of the theory as $\left\langle T_{\mu}^{\mu}\right\rangle=c W^{2}-a E_{4}+\cdots$, where $W$ is the Weyl tensor, $E_{4}$ is the Euler density, and ellipses denote operators constructed from the fields in the theory. A function related to the CFT function $a$, often denoted $\tilde{a}$, was proven to be monotonically decreasing following the RG flow from a UV fixed point 
to an IR one $[26,28]$. In fact, the RG flow of the $\tilde{a}$-function is related to the dynamics by means of the $\beta$-functions of the theory; it is given by

$$
\mu \frac{\partial \tilde{a}}{\partial \mu}=-\chi_{i j} \beta^{i} \beta^{j}
$$

where $\chi_{i j}$ is known as the Zamolodchikov metric. Evaluated at a fixed point, $\tilde{a}$ reduces to the $a$-function.

In all of the models studied in this paper there is only a UV fixed point present, whereas dynamics in the IR is not known. Nevertheless, central charges of the UV fixed points can still be used to test whether the fixed points are reliable.

In any CFT, both $a$ and $c$ have to be positive, and their ratio has to satisfy the so-called collider bounds [66], namely

$$
\frac{1}{3} \leq\left.\frac{a}{c}\right|_{\mathrm{FP}} \leq \frac{31}{18}
$$

In perturbation theory, central charges are expanded in series

$$
\begin{aligned}
& \tilde{a}=\tilde{a}_{\text {free }}+\frac{\tilde{a}^{(1)}}{(4 \pi)^{2}}+\frac{\tilde{a}^{(2)}}{(4 \pi)^{4}}+\ldots \\
& c=c_{\text {free }}+\frac{c^{(1)}}{(4 \pi)^{4}}+\ldots
\end{aligned}
$$

and since free-field theory contributions are positive [67],

$$
\begin{aligned}
\tilde{a}_{\text {free }} & =\frac{1}{(4 \pi)^{2}} \frac{n_{s}+11 / 2 n_{w}+62 n_{v}}{360} \\
c_{\text {free }} & =\frac{1}{(4 \pi)^{2}} \frac{1 / 6 n_{s}+n_{w}+2 n_{v}}{20}
\end{aligned}
$$

$\left(n_{s}, n_{w}\right.$, and $n_{v}$ referring to scalar, Weyl and vector degrees of freedom, respectively), the positivity of the CFT functions is ensured in perturbation theory.

There is a correlation between critical exponents and the change in central charges, which for the $a$-function can be explained as follows. At the fixed point we have,

$$
\tilde{a}^{*}=a^{*}=a_{\text {free }}+\frac{1}{4} \sum_{i} b_{i} \chi_{g_{i} g_{i}} \alpha_{i}^{*}\left(1+A_{i} \alpha_{i}^{*}\right)
$$

where $i$ runs over simple gauge groups, $b_{1}=B_{1}, b_{2}=-B_{2}, b_{3}=-B_{3}$ are the one-loop coefficients of the gauge beta functions, and $\chi_{g_{i} g_{i}}$ and $A_{i}$ are components of the Zamolodchikov metric, see [56]. One-loop critical exponent follows from $\beta_{i}= \pm B_{i} \alpha_{i}^{2}$ ( + for the group $\mathrm{U}(1),-$ otherwise), and reads $\theta^{1 L}=2 b_{i} \alpha_{i}^{*}$. Then,

$$
\delta a=\frac{a^{*}-a_{\mathrm{free}}}{a_{\mathrm{free}}}=\frac{1}{8 a_{\mathrm{free}}} \sum_{i} \theta_{i}^{1 L} \chi_{g_{i} g_{i}}\left(1+A_{i} \alpha_{i}^{*}\right),
$$

which explains the correlation. 


\section{All the fixed points in the 210 approximation scheme}

In table 12 we list all the distinct zeroes of the $\beta$-functions in the 210 approximation scheme for all the models discussed in the text and for the SM. There are altogether 32 zeroes, with the Gaussian fixed point appearing with multiplicity four (this is the reason for missing fixed point $P_{20}, P_{27}, P_{32}$, which are copies of $P_{1}$ ).

The column labelled by $N_{f}=0$ contains the values of $\alpha_{1}^{*}, \alpha_{2}^{*}, \alpha_{3}^{*}, \alpha_{t}^{*}$ for the matter content of the SM (the coupling $\alpha_{y}^{*}$ does not appear in the SM). In this case the fixed points all come in pairs. When $N_{f} \neq 0$ this degeneracy is lifted and all the fixed points are different.

Note that the fixed points can be roughly divided in two classes. The fixed points with $\alpha_{1}^{*}=0$ have coordinates $\alpha_{i}^{*}$ independent of $Y$. The remaining fixed points have coordinates that in general depend on all the quantum numbers.

\section{Coefficients of the NLO and NNLO $\beta$-functions}

The $\beta$-function in eqs. (4.7)-(4.9) contain a number of coefficients that we collect in this appendix. The BSM fermions enter in the running of $\alpha_{t}$ via the coefficients

$$
B_{t 1}=Y^{2} N_{f} d_{R_{2}} d_{R_{3}}, B_{t 2}=S_{R_{2}} N_{f} d_{R_{3}}, B_{t 3}=S_{R_{3}} N_{f} d_{R_{2}} .
$$

For the BSM Yukawa coupling, besides the terms in eq. (4.6), we have the coefficients

$$
\begin{aligned}
V & =\frac{1}{2} N_{f}^{2}+3 N_{f} d_{R_{2}} d_{R_{3}}, & V_{1} & =2\left(8 N_{f}+5 d_{R_{2}} d_{R_{3}}\right) Y^{2}, \\
V_{2} & =2\left(8 N_{f}+5 d_{R_{2}} d_{R_{3}}\right) C_{R_{2}}, & V_{3} & =2\left(8 N_{f}+5 d_{R_{2}} d_{R_{3}}\right) C_{R_{3}}, \\
W_{1} & =\left(\frac{211}{3}-6 Y^{2}+\frac{40}{3} Y^{2} N_{f} d_{R_{2}} d_{R_{3}}\right) Y^{2}, & W_{12} & =12 Y^{2} C_{R_{2}}, \\
W_{2} & =\left(-\frac{257}{3}-6 C_{R_{2}}+\frac{40}{3} N_{f} S_{R_{2}} d_{R_{3}}\right) C_{R_{2}}, & W_{23} & =12 C_{R_{2}} C_{R_{3}}, \\
W_{3} & =\left(-154-6 C_{R_{3}}+\frac{40}{3} N_{f} S_{R_{3}} d_{R_{2}}\right) C_{R_{3}} & W_{13} & =12 Y^{2} C_{R_{3} .} .
\end{aligned}
$$

The gauge $\beta$-functions get more contributions. These are split in two classes: the Yukawa contributions:

$$
\begin{aligned}
K_{y 1} & =6 Y^{2} N_{f}^{3} d_{R_{2}} d_{R_{3}}+7 Y^{2} N_{f}^{2} d_{R_{2}}^{2} d_{R_{3}}^{2}, & K_{11} & =6 Y^{4} N_{f}^{2} d_{R_{2}} d_{R_{3}}, \\
K_{12} & =6 Y^{2} C_{R_{2}} N_{f}^{2} d_{R_{2}} d_{R_{3}}, & K_{13} & =6 Y^{2} C_{R_{3}} N_{f}^{2} d_{R_{2}} d_{R_{3}}, \\
K_{y 2} & =2 C_{R_{2}} N_{f}^{3} d_{R_{2}} d_{R_{3}}+\frac{7}{3} C_{R_{2}} N_{f}^{2} d_{R_{2}}^{2} d_{R_{3}}^{2}, & K_{21} & =2 Y^{2} C_{R_{2}} N_{f}^{2} d_{R_{2}} d_{R_{3}}, \\
K_{22} & =16 C_{R_{2}} N_{f}^{2} d_{R_{2}} d_{R_{3}}+2 C_{R_{2}}^{2} N_{f}^{2} d_{R_{2}} d_{R_{3}}, & K_{23} & =2 C_{R_{2}} C_{R_{3}} N_{f}^{2} d_{R_{2}} d_{R_{3}}, \\
K_{y 3} & =\frac{3}{4} C_{R_{3}} N_{f}^{3} d_{R_{2}} d_{R_{3}}+\frac{7}{8} C_{R_{3}} N_{f}^{2} d_{R_{2}}^{2} d_{R_{3}}^{2}, & K_{31} & =\frac{3}{4} Y^{2} C_{R_{3}} N_{f}^{2} d_{R_{2}} d_{R_{3}}, \\
K_{33} & =9 C_{R_{3}} N_{f}^{2} d_{R_{2}} d_{R_{3}}+\frac{3}{4} C_{R_{3}}^{2} N_{f}^{2} d_{R_{2}} d_{R_{3}} & K_{32} & =\frac{3}{4} C_{R_{2}} C_{R_{3}} N_{f}^{2} d_{R_{2}} d_{R_{3}},
\end{aligned}
$$




\begin{tabular}{|c|c|c|c|c|c|c|}
\hline & $\alpha_{1}^{*}$ & $\alpha_{2}^{*}$ & $\alpha_{3}^{*}$ & $\alpha_{t}^{*}$ & $\alpha_{y}^{*}$ & $N_{f}=0$ \\
\hline$P_{1}$ & 0 & 0 & 0 & 0 & 0 & $(0,0,0,0)$ \\
\hline$P_{2}$ & 0 & $\alpha_{2}^{*}(p, q, \ell)$ & $\alpha_{3}^{*}(p, q, \ell)$ & 0 & $\alpha_{y}^{*}(p, q, \ell)$ & $\left(0, \frac{499}{617},-\frac{319}{2468}, 0\right)$ \\
\hline$P_{3}$ & 0 & $\alpha_{2}^{*}(p, q, \ell)$ & $\alpha_{3}^{*}(p, q, \ell)$ & $\alpha_{t}^{*}(p, q, \ell)$ & $\alpha_{y}^{*}(p, q, \ell)$ & $\left(0, \frac{1226}{1411},-\frac{189}{1411}, \frac{277}{1411}\right)$ \\
\hline$P_{4}$ & 0 & $\alpha_{2}^{*}(p, q, \ell)$ & $\alpha_{3}^{*}(p, q, \ell)$ & 0 & 0 & $\left(0, \frac{499}{617},-\frac{319}{2468}, 0\right)$ \\
\hline$P_{5}$ & 0 & $\alpha_{2}^{*}(p, q, \ell)$ & $\alpha_{3}^{*}(p, q, \ell)$ & $\alpha_{t}^{*}(p, q, \ell)$ & 0 & $\left(0, \frac{1226}{1411},-\frac{189}{1411}, \frac{277}{1411}\right)$ \\
\hline$P_{6}$ & $\alpha_{1}^{*}(p, q, \ell, Y)$ & $\alpha_{2}^{*}(p, q, \ell, Y)$ & $\alpha_{3}^{*}(p, q, \ell, Y)$ & 0 & $\alpha_{y}^{*}(p, q, \ell, Y)$ & $\left(-\frac{7938}{9257}, \frac{9841}{9257},-\frac{5395}{37028}, 0\right)$ \\
\hline$P_{7}$ & $\alpha_{1}^{*}(p, q, \ell, Y)$ & $\alpha_{2}^{*}(p, q, \ell, Y)$ & $\alpha_{3}^{*}(p, q, \ell, Y)$ & $\alpha_{t}^{*}(p, q, \ell, Y)$ & $\alpha_{y}^{*}(p, q, \ell, Y)$ & $\left(-\frac{121821}{142153}, \frac{151229}{142153},-\frac{41441}{284306}, \frac{427}{142153}\right)$ \\
\hline$P_{8}$ & 0 & 0 & $\alpha_{3}^{*}(p, q, \ell)$ & $\alpha_{t}^{*}(p, q, \ell)$ & $\alpha_{y}^{*}(p, q, \ell)$ & $\left(0,0,-\frac{9}{38},-\frac{8}{19}\right)$ \\
\hline$P_{9}$ & 0 & 0 & $\alpha_{3}^{*}(p, q, \ell)$ & 0 & $\alpha_{y}^{*}(p, q, \ell)$ & $\left(0,0,-\frac{7}{26}, 0\right)$ \\
\hline$P_{10}$ & 0 & 0 & $\alpha_{3}^{*}(p, q, \ell)$ & $\alpha_{t}^{*}(p, q, \ell)$ & 0 & $\left(0,0,-\frac{9}{38},-\frac{8}{19}\right)$ \\
\hline$P_{11}$ & 0 & 0 & $\alpha_{3}^{*}(p, q, \ell)$ & 0 & 0 & $\left(0,0,-\frac{7}{26}, 0\right)$ \\
\hline$P_{12}$ & $\alpha_{1}^{*}(p, q, \ell, Y)$ & 0 & $\alpha_{3}^{*}(p, q, \ell, Y)$ & 0 & $\alpha_{y}^{*}(p, q, \ell, Y)$ & $\left(-\frac{225}{943}, 0,-\frac{1079}{3772}\right)$ \\
\hline$P_{13}$ & $\alpha_{1}^{*}(p, q, \ell, Y)$ & 0 & $\alpha_{3}^{*}(p, q, \ell, Y)$ & $\alpha_{t}^{*}(p, q, \ell, Y)$ & $\alpha_{y}^{*}(p, q, \ell, Y)$ & $\left(-\frac{7266}{16847}, 0,-\frac{4286}{16847},-\frac{9907}{16847}\right)$ \\
\hline$P_{14}$ & $\alpha_{1}^{*}(p, q, \ell, Y)$ & 0 & $\alpha_{3}^{*}(p, q, \ell, Y)$ & 0 & 0 & $\left(-\frac{225}{943}, 0,-\frac{1079}{3772}\right)$ \\
\hline$P_{15}$ & $\alpha_{1}^{*}(p, q, \ell, Y)$ & 0 & $\alpha_{3}^{*}(p, q, \ell, Y)$ & $\alpha_{t}^{*}(p, q, \ell, Y)$ & 0 & $\left(-\frac{7266}{16847}, 0,-\frac{4286}{16847},-\frac{9907}{16847}\right)$ \\
\hline$P_{16}$ & 0 & $\alpha_{2}^{*}(p, q, \ell)$ & 0 & 0 & $\alpha_{y}^{*}(p, q, \ell)$ & $\left(0, \frac{19}{35}, 0,0\right)$ \\
\hline$P_{17}$ & 0 & $\alpha_{2}^{*}(p, q, \ell)$ & 0 & $\alpha_{t}^{*}(p, q, \ell)$ & $\alpha_{y}^{*}(p, q, \ell)$ & $\left(0, \frac{38}{61}, 0, \frac{19}{61}\right)$ \\
\hline$P_{18}$ & 0 & $\alpha_{2}^{*}(p, q, \ell)$ & 0 & 0 & 0 & $\left(0, \frac{19}{35}, 0,0\right)$ \\
\hline$P_{19}$ & 0 & $\alpha_{2}^{*}(p, q, \ell)$ & 0 & $\alpha_{t}^{*}(p, q, \ell)$ & 0 & $\left(0, \frac{38}{61}, 0, \frac{19}{61}\right)$ \\
\hline$P_{21}$ & $\alpha_{1}^{*}(p, q, \ell, Y)$ & 0 & 0 & 0 & 0 & $\left(-\frac{123}{199}, 0,0,0\right)$ \\
\hline$P_{22}$ & $\alpha_{1}^{*}(p, q, \ell, Y)$ & 0 & 0 & $\alpha_{t}^{*}(p, q, \ell, Y)$ & 0 & $\left(-\frac{2214}{3293}, 0,0,-\frac{697}{3293}\right)$ \\
\hline$P_{23}$ & $\alpha_{1}^{*}(p, q, \ell, Y)$ & 0 & 0 & $\alpha_{t}^{*}(p, q, \ell, Y)$ & $\alpha_{y}^{*}(p, q, \ell, Y)$ & $\left(-\frac{2214}{3293}, 0,0,-\frac{697}{3293}\right)$ \\
\hline$P_{24}$ & $\alpha_{1}^{*}(p, q, \ell, Y)$ & 0 & 0 & 0 & $\alpha_{y}^{*}(p, q, \ell, Y)$ & $\left(-\frac{123}{199}, 0,0,0\right)$ \\
\hline$P_{25}$ & $\alpha_{1}^{*}(p, q, \ell, Y)$ & $\alpha_{2}^{*}(p, q, \ell, Y)$ & 0 & 0 & $\alpha_{y}^{*}(p, q, \ell, Y)$ & $\left(-\frac{1461}{1559}, \frac{1222}{1559}, 0,0\right)$ \\
\hline$P_{26}$ & $\alpha_{1}^{*}(p, q, \ell, Y)$ & $\alpha_{2}^{*}(p, q, \ell, Y)$ & 0 & $\alpha_{t}^{*}(p, q, \ell, Y)$ & $\alpha_{y}^{*}(p, q, \ell, Y)$ & $\left(-\frac{21627}{23569}, \frac{515}{637}, 0, \frac{2719}{23569}\right)$ \\
\hline$P_{28}$ & $\alpha_{1}^{*}(p, q, \ell, Y)$ & $\alpha_{2}^{*}(p, q, \ell, Y)$ & 0 & 0 & 0 & $\left(-\frac{1461}{1559}, \frac{1222}{1559}, 0,0\right)$ \\
\hline$P_{29}$ & $\alpha_{1}^{*}(p, q, \ell, Y)$ & $\alpha_{2}^{*}(p, q, \ell, Y)$ & 0 & $\alpha_{t}^{*}(p, q, \ell, Y)$ & 0 & $\left(-\frac{21627}{23569}, \frac{515}{637}, 0, \frac{2719}{23569}\right)$ \\
\hline$P_{30}$ & $\alpha_{1}^{*}(p, q, \ell, Y)$ & $\alpha_{2}^{*}(p, q, \ell, Y)$ & $\alpha_{3}^{*}(p, q, \ell, Y)$ & 0 & 0 & $\left(-\frac{7938}{9257}, \frac{9841}{9257},-\frac{5395}{37028}, 0\right)$ \\
\hline$P_{31}$ & $\alpha_{1}^{*}(p, q, \ell, Y)$ & $\alpha_{2}^{*}(p, q, \ell, Y)$ & $\alpha_{3}^{*}(p, q, \ell, Y)$ & $\alpha_{t}^{*}(p, q, \ell, Y)$ & 0 & $\left(-\frac{121821}{142153}, \frac{151229}{142153},-\frac{41441}{284306}, \frac{427}{142153}\right)$ \\
\hline
\end{tabular}

Table 12. List of all the fixed points in the 210 approximation scheme. When non-zero, the dependence on the quantum numbers is indicated. Only the highlighted fixed points appear in the tables in the main text. The column $N_{f}=0$ contains the values for the SM. 
and the gauge contributions, which contain the diagonal terms

$$
\begin{aligned}
M_{11}= & \frac{388613}{2592}+\frac{4405}{162} N_{f} Y^{2} d_{R_{2}} d_{R_{3}}+\frac{463}{9} N_{f} Y^{4} d_{R_{2}} d_{R_{3}} \\
& +4 N_{f} Y^{6} d_{R_{2}} d_{R_{3}}+\frac{88}{9} N_{f}^{2} Y^{6} d_{R_{2}}^{2} d_{R_{3}}^{2}, \\
M_{22}= & \frac{324953}{864}+\frac{13411}{54} N_{f} S_{R_{2}} d_{R_{3}}+\frac{533}{9} N_{f} C_{R_{2}} S_{R_{2}} d_{R_{3}}-4 N_{f} C_{R_{2}}^{2} S_{R_{2}} d_{R_{3}} \\
& -\frac{632}{27} N_{f}^{2} S_{R_{2}}^{2} d_{R_{3}}^{2}-\frac{88}{9} C_{R_{2}} N_{f}^{2} S_{R_{2}}^{2} d_{R_{3}}^{2}, \\
M_{33}= & 65+\frac{6242}{9} N_{f} S_{R_{3}} d_{R_{2}}+\frac{322}{3} N_{f} C_{R_{3}} S_{R_{3}} d_{R_{2}}-4 N_{f} C_{R_{3}}^{2} S_{R_{3}} d_{R_{2}} \\
& -\frac{316}{9} N_{f}^{2} S_{R_{3}}^{2} d_{R_{2}}^{2}-\frac{88}{9} C_{R_{3}} N_{f}^{2} S_{R_{3}}^{2} d_{R_{2}}^{2},
\end{aligned}
$$

as well as mixed coefficients

$$
\begin{aligned}
& M_{12}=\frac{205}{48}-8 C_{R_{2}} N_{f} Y^{4} d_{R_{2}} d_{R_{3}}, \\
& M_{13}=\frac{274}{27}+8 C_{R_{3}} N_{f} Y^{4} d_{R_{2}} d_{R_{3}}, \\
& M_{21}=\frac{291}{16}+32 Y^{2} N_{f} S_{R_{2}} d_{R_{3}}-8 Y^{2} C_{R_{2}} N_{f} S_{R_{2}} d_{R_{3}}, \\
& M_{23}=78+32 C_{R_{3}} N_{f} S_{R_{2}} d_{R_{3}}-8 C_{R_{2}} C_{R_{3}} N_{f} S_{R_{2}} d_{R_{3}}, \\
& M_{31}=\frac{154}{9}+48 Y^{2} N_{f} S_{R_{3}} d_{R_{2}}-8 Y^{2} C_{R_{3}} N_{f} S_{R_{3}} d_{R_{2}}, \\
& M_{32}=42+48 C_{R_{2}} N_{f} S_{R_{3}} d_{R_{2}}-8 C_{R_{2}} C_{R_{3}} N_{f} S_{R_{3}} d_{R_{2}}, \\
& G_{23}=2+8 C_{R_{2}} C_{R_{3}} N_{f} Y^{2} d_{R_{2}} d_{R_{3}} \text {, } \\
& G_{13}=\frac{2}{3}+8 Y^{2} C_{R_{3}} N_{f} S_{R_{2}} d_{R_{3}} \text {, } \\
& G_{12}=\frac{1}{4}+8 Y^{2} C_{R_{2}} N_{f} S_{R_{3}} d_{R_{2}}, \\
& H_{11}=\frac{1315}{32}+\frac{245}{9} C_{R_{2}} N_{f} Y^{2} d_{R_{2}} d_{R_{3}}-4 C_{R_{2}}^{2} N_{f} Y^{2} d_{R_{2}} d_{R_{3}}+\frac{23}{2} N_{f} S_{R_{2}} d_{R_{3}} \\
& -\frac{88}{9} C_{R_{2}} N_{f}^{2} Y^{2} S_{R_{2}} d_{R_{2}} d_{R_{3}}^{2} \\
& G_{11}=198+\frac{178}{3} C_{R_{3}} N_{f} Y^{2} d_{R_{2}} d_{R_{3}}-4 C_{R_{3}}^{2} N_{f} Y^{2} d_{R_{2}} d_{R_{3}}-\frac{968}{27} N_{f} S_{R_{3}} d_{R_{2}} \\
& -\frac{88}{9} C_{R_{3}} N_{f}^{2} Y^{2} S_{R_{3}} d_{R_{2}}^{2} d_{R_{3}} \text {, } \\
& H_{22}=\frac{5597}{288}+\frac{23}{6} N_{f} Y^{2} d_{R_{2}} d_{R_{3}}+\frac{463}{9} Y^{2} N_{f} S_{R_{2}} d_{R_{3}}+4 N_{f} Y^{4} S_{R_{2}} d_{R_{3}} \\
& +\frac{88}{9} N_{f}^{2} Y^{4} S_{R_{2}} d_{R_{2}} d_{R_{3}}^{2}, \\
& G_{22}=162+\frac{178}{3} C_{R_{3}} N_{f} S_{R_{2}} d_{R_{3}}-4 C_{R_{3}}^{2} N_{f} S_{R_{2}} d_{R_{3}}-\frac{88}{3} N_{f} S_{R_{3}} d_{R_{2}} \\
& -\frac{88}{9} C_{R_{3}} N_{f}^{2} S_{R_{2}} S_{R_{3}} d_{R_{2}} d_{R_{3}}
\end{aligned}
$$




$$
\begin{aligned}
H_{33}= & \frac{2615}{108}+\frac{121}{27} N_{f} Y^{2} d_{R_{2}} d_{R_{3}}+\frac{463}{9} Y^{2} N_{f} S_{R_{3}} d_{R_{2}}+4 N_{f} Y^{4} S_{R_{3}} d_{R_{2}} \\
& +\frac{88}{9} N_{f}^{2} Y^{4} S_{R_{3}} d_{R_{3}} d_{R_{2}}^{2}, \\
G_{33}= & \frac{109}{4}-11 N_{f} S_{R_{2}} d_{R_{3}}+\frac{245}{9} C_{R_{2}} N_{f} S_{R_{3}} d_{R_{2}}-4 C_{R_{2}}^{2} N_{f} S_{R_{3}} d_{R_{2}} \\
& -\frac{88}{9} C_{R_{2}} N_{f}^{2} S_{R_{2}} S_{R_{3}} d_{R_{2}} d_{R_{3}},
\end{aligned}
$$

Open Access. This article is distributed under the terms of the Creative Commons Attribution License (CC-BY 4.0), which permits any use, distribution and reproduction in any medium, provided the original author(s) and source are credited.

\section{References}

[1] K.G. Wilson, Renormalization group and critical phenomena. 1. Renormalization group and the Kadanoff scaling picture, Phys. Rev. B 4 (1971) 3174 [InSPIRE].

[2] S. Weinberg, Ultraviolet divergences in quantum theories of gravitation, in General relativity: an Einstein centenary survey, S.W. Hawking and W. Israel, Cambridge University Press, Cambridge U.K. (2010).

[3] H. Gies and M.M. Scherer, Asymptotic safety of simple Yukawa systems, Eur. Phys. J. C 66 (2010) 387 [arXiv:0901.2459] [inSPIRE].

[4] H. Gies, S. Rechenberger and M.M. Scherer, Towards an asymptotic-safety scenario for chiral Yukawa systems, Eur. Phys. J. C 66 (2010) 403 [arXiv:0907.0327] [InSPIRE].

[5] M. Fabbrichesi, R. Percacci, A. Tonero and O. Zanusso, Asymptotic safety and the gauged $\mathrm{SU}(N)$ nonlinear $\sigma$-model, Phys. Rev. D 83 (2011) 025016 [arXiv:1010.0912] [INSPIRE].

[6] F. Bazzocchi et al., Fermions and Goldstone bosons in an asymptotically safe model, Phys. Lett. B 705 (2011) 388 [arXiv:1105.1968] [INSPIRE].

[7] M. Fabbrichesi, R. Percacci, A. Tonero and L. Vecchi, The electroweak $S$ and T parameters from a fixed point condition, Phys. Rev. Lett. 107 (2011) 021803 [arXiv:1102.2113] [INSPIRE].

[8] H. Gies, S. Rechenberger, M.M. Scherer and L. Zambelli, An asymptotic safety scenario for gauged chiral Higgs-Yukawa models, Eur. Phys. J. C 73 (2013) 2652 [arXiv:1306.6508] [INSPIRE].

[9] D.F. Litim and F. Sannino, Asymptotic safety guaranteed, JHEP 12 (2014) 178 [arXiv: 1406.2337] [INSPIRE].

[10] A.D. Bond and D.F. Litim, Theorems for asymptotic safety of gauge theories, Eur. Phys. J. C 77 (2017) 429 [Erratum ibid. C 77 (2017) 525] [arXiv: 1608.00519] [INSPIRE].

[11] D.F. Litim, M. Mojaza and F. Sannino, Vacuum stability of asymptotically safe gauge-Yukawa theories, JHEP 01 (2016) 081 [arXiv: 1501.03061] [INSPIRE].

[12] B. Bajc and F. Sannino, Asymptotically safe grand unification, JHEP 12 (2016) 141 [arXiv: 1610.09681] [INSPIRE].

[13] S. Abel and F. Sannino, Radiative symmetry breaking from interacting UV fixed points, Phys. Rev. D 96 (2017) 056028 [arXiv: 1704.00700] [INSPIRE]. 
[14] S. Abel and F. Sannino, Framework for an asymptotically safe Standard Model via dynamical breaking, Phys. Rev. D 96 (2017) 055021 [arXiv:1707.06638] [InSPIRE].

[15] A.D. Bond, G. Hiller, K. Kowalska and D.F. Litim, Directions for model building from asymptotic safety, JHEP 08 (2017) 004 [arXiv: 1702.01727] [INSPIRE].

[16] R. Mann et al., Asymptotically safe standard model via vectorlike fermions, Phys. Rev. Lett. 119 (2017) 261802 [arXiv:1707.02942] [INSPIRE].

[17] G.M. Pelaggi et al., Asymptotically safe standard model extensions?, Phys. Rev. D 97 (2018) 095013 [arXiv: 1708.00437] [INSPIRE].

[18] G.M. Pelaggi, F. Sannino, A. Strumia and E. Vigiani, Naturalness of asymptotically safe Higgs, Front. in Phys. 5 (2017) 49 [arXiv:1701.01453] [INSPIRE].

[19] S. Ipek, A.D. Plascencia and J. Turner, Assessing perturbativity and vacuum stability in high-scale leptogenesis, arXiv:1806.00460 [INSPIRE].

[20] M. Gell-Mann and F.E. Low, Quantum electrodynamics at small distances, Phys. Rev. 95 (1954) 1300 [INSPIRE].

[21] M. Gockeler et al., Is there a Landau pole problem in QED?, Phys. Rev. Lett. 80 (1998) 4119 [hep-th/9712244] [INSPIRE].

[22] G. Degrassi et al., Higgs mass and vacuum stability in the Standard Model at NNLO, JHEP 08 (2012) 098 [arXiv: 1205.6497] [INSPIRE].

[23] T.J. Hollowood, Renormalization group and fixed points in quantum field theory, Springer Briefs in Physics, Springer, Germany (2013).

[24] D.G.C. McKeon and C. Zhao, Multiple couplings and renormalization scheme ambiguities, Nucl. Phys. B 932 (2018) 425 [arXiv: 1711.04758] [INSPIRE].

[25] A. Codello, M. Safari, G.P. Vacca and O. Zanusso, Functional perturbative RG and CFT data in the $\epsilon$-expansion, Eur. Phys. J. C 78 (2018) 30 [arXiv:1705.05558] [inSPIRE].

[26] H. Osborn, Derivation of a four dimensional c-theorem for renormaliseable quantum field theories, Phys. Lett. B222 (1989) 97.

[27] J.L. Cardy, Is there a c-theorem in four dimensions?, Phys. Lett. B 215 (1988) 749.

[28] I. Jack and H. Osborn, Analogs of the c-theorem for four-dimensional renormalisable field theories, Nucl. Phys. B 343 (1990) 647.

[29] H. Osborn, Weyl consistency conditions and a local renormalisation group equation for general renormalisable field theories, Nucl. Phys. B 363 (1991) 486.

[30] O. Antipin et al., Standard Model vacuum stability and Weyl consistency conditions, JHEP 08 (2013) 034 [arXiv: 1306.3234] [INSPIRE].

[31] A.D. Bond, D.F. Litim, G. Medina Vazquez and T. Steudtner, UV conformal window for asymptotic safety, Phys. Rev. D 97 (2018) 036019 [arXiv:1710.07615] [INSPIRE].

[32] D.J. Gross and F. Wilczek, Ultraviolet behavior of nonabelian gauge theories, Phys. Rev. Lett. 30 (1973) 1343 [INSPIRE].

[33] H.D. Politzer, Reliable perturbative results for strong interactions?, Phys. Rev. Lett. 30 (1973) 1346 [INSPIRE].

[34] T.P. Cheng, E. Eichten and L.-F. Li, Higgs phenomena in asymptotically free gauge theories, Phys. Rev. D 9 (1974) 2259 [inSPIRE]. 
[35] D.J. Gross and F. Wilczek, Asymptotically free gauge theories - I, Phys. Rev. D 8 (1973) 3633 [INSPIRE].

[36] W.E. Caswell, Asymptotic behavior of nonabelian gauge theories to two loop order, Phys. Rev. Lett. 33 (1974) 244 [INSPIRE].

[37] O.V. Tarasov and A.A. Vladimirov, Two loop renormalization of the Yang-Mills theory in an arbitrary gauge, Sov. J. Nucl. Phys. 25 (1977) 585 [Yad. Fiz. 25 (1977) 1104] [INSPIRE].

[38] D.R.T. Jones, The two loop $\beta$-function for a $G_{1} \times G_{2}$ gauge theory, Phys. Rev. D 25 (1982) 581 [INSPIRE].

[39] M.E. Machacek and M.T. Vaughn, Two loop renormalization group equations in a general quantum field theory. 1. Wave function renormalization, Nucl. Phys. B 222 (1983) 83 [INSPIRE].

[40] M. Fischler and J. Oliensis, Two loop corrections to the evolution of the Higgs-Yukawa coupling constant, Phys. Lett. B 119 (1982) 385.

[41] M.E. Machacek and M.T. Vaughn, Two loop renormalization group equations in a general quantum field theory. 2. Yukawa couplings, Nucl. Phys. B 236 (1984) 221 [inSPIRE].

[42] I. Jack and H. Osborn, Background field calculations in curved space-time. 1. General formalism and application to scalar fields, Nucl. Phys. B 234 (1984) 331 [INSPIRE].

[43] M.E. Machacek and M.T. Vaughn, Two loop renormalization group equations in a general quantum field theory. 3. Scalar quartic couplings, Nucl. Phys. B 249 (1985) 70 [INSPIRE].

[44] C. Ford, I. Jack and D.R.T. Jones, The standard model effective potential at two loops, Nucl. Phys. B 387 (1992) 373 [Erratum ibid. B 504 (1997) 551] [hep-ph/0111190] [INSPIRE].

[45] H. Arason et al., Renormalization group study of the standard model and its extensions. 1. The Standard model, Phys. Rev. D 46 (1992) 3945 [inSPIRE].

[46] M.-x. Luo, H.-w. Wang and Y. Xiao, Two loop renormalization group equations in general gauge field theories, Phys. Rev. D 67 (2003) 065019 [hep-ph/0211440] [INSPIRE].

[47] M.-x. Luo and Y. Xiao, Two loop renormalization group equations in the standard model, Phys. Rev. Lett. 90 (2003) 011601 [hep-ph/0207271] [INSPIRE].

[48] T. Curtright, Three loop charge renormalization effects due to quartic scalar selfinteractions, Phys. Rev. D 21 (1980) 1543 [INSPIRE].

[49] A.G.M. Pickering, J.A. Gracey and D.R.T. Jones, Three loop gauge $\beta$-function for the most general single gauge coupling theory, Phys. Lett. B 510 (2001) 347 [Erratum ibid. B 535 (2002) 377] [hep-ph/0104247] [INSPIRE].

[50] L.N. Mihaila, J. Salomon and M. Steinhauser, Gauge coupling $\beta$-functions in the Standard Model to three loops, Phys. Rev. Lett. 108 (2012) 151602 [arXiv:1201.5868] [INSPIRE].

[51] L. Mihaila, Three-loop gauge beta function in non-simple gauge groups, PoS (RADCOR 2013) 060 .

[52] L. Mihaila, to appear.

[53] K.G. Chetyrkin and M.F. Zoller, Three-loop $\beta$-functions for top-Yukawa and the Higgs self-interaction in the Standard Model, JHEP 06 (2012) 033 [arXiv:1205.2892] [INSPIRE].

[54] A.V. Bednyakov, A.F. Pikelner and V.N. Velizhanin, Yukawa coupling $\beta$-functions in the Standard Model at three loops, Phys. Lett. B 722 (2013) 336 [arXiv:1212.6829] [INSPIRE]. 
[55] A.V. Bednyakov, A.F. Pikelner and V.N. Velizhanin, Higgs self-coupling $\beta$-function in the Standard Model at three loops, Nucl. Phys. B 875 (2013) 552 [arXiv:1303.4364] [InSPIRE].

[56] N.A. Dondi, V. Prochazka and F. Sannino, Conformal data of fundamental gauge-yukawa theories, Phys. Rev. D 98 (2018) 045002 [arXiv:1712.05388] [INSPIRE].

[57] A.D. Bond, D.F. Litim, G. Medina Vazquez and T. Steudtner, UV conformal window for asymptotic safety, Phys. Rev. D 97 (2018) 036019 [arXiv:1710.07615] [INSPIRE].

[58] B. Holdom, Large $N$ flavor $\beta$-functions: a recap, Phys. Lett. B 694 (2011) 74 [arXiv: 1006.2119] [INSPIRE].

[59] M. Cirelli, N. Fornengo and A. Strumia, Minimal dark matter, Nucl. Phys. B 753 (2006) 178 [hep-ph/0512090] [INSPIRE].

[60] E.W. Kolb, D.J.H. Chung and A. Riotto, WIMPzillas!, AIP Conf. Proc. 484 (1999) 91 [hep-ph/9810361] [INSPIRE].

[61] E. Molinaro, F. Sannino and Z.W. Wang, Safe Pati-Salam, arXiv:1807.03669 [INSPIRE].

[62] U. Harst and M. Reuter, QED coupled to QEG, JHEP 05 (2011) 119 [arXiv:1101.6007] [INSPIRE].

[63] N. Christiansen and A. Eichhorn, An asymptotically safe solution to the U(1) triviality problem, Phys. Lett. B 770 (2017) 154 [arXiv:1702.07724] [INSPIRE].

[64] P. Donà, A. Eichhorn and R. Percacci, Matter matters in asymptotically safe quantum gravity, Phys. Rev. D 89 (2014) 084035 [arXiv:1311.2898] [InSPIRE].

[65] A. Eichhorn and A. Held, Top mass from asymptotic safety, Phys. Lett. B 777 (2018) 217 [arXiv: 1707.01107] [INSPIRE].

[66] D.M. Hofman and J. Maldacena, Conformal collider physics: Energy and charge correlations, JHEP 05 (2008) 012 [arXiv: 0803.1467] [INSPIRE].

[67] M.J. Duff, Observations on conformal anomalies, Nucl. Phys. B 125 (1977) 334 [inSPIRE]. 